\title{
Strategic Reasoning in Persuasion Games: An EXPERIMENT*
}

\author{
Ying Xue $\mathrm{Li}^{\dagger} \quad$ Burkhard C. Schipper ${ }^{\ddagger}$
}

February 8, 2020

\begin{abstract}
We study experimentally persuasion games in which a sender (e.g., a seller) with private information provides verifiable but potentially vague information (e.g., about the quality of a product) to a receiver (e.g., a buyer). Various theoretical solution concepts such as sequential equilibrium or iterated admissibility predict unraveling of information. Iterated admissibility also provides predictions for every finite level of reasoning about rationality. Overall we observe behavior consistent with relatively high levels of reasoning. While iterated admissibility implies that the level of reasoning required for unraveling is increasing in the number of quality levels, we find only insignificantly more unraveling in a game with two quality levels compared to a game with four quality levels. There is weak evidence for learning. Participants display difficulties in transferring learning to unravel in a game with two quality levels to a game with four quality levels. Finally, participants who score higher on cognitive abilities in Raven's progressive matrices test also display significantly higher levels of reasoning in our persuasion games although the effect-size is small.
\end{abstract}

Keywords: Persuasion games, verifiable information, communication, disclosure, unraveling, iterated admissibility, prudent rationalizability, common strong cautious belief in rationality, level- $k$ reasoning, experiments, cognitive ability.

JEL-Classification: C72, C92, D82, D83.

${ }^{*}$ We thank the editor, associate editor, three anonymous reviewers, Joe Sobel, Daniel Martin, and Eduardo Perez-Richet as well as participants in the 6th Xiamen University International Workshop on Experimental Economics 2016, the 2018 Bay Area Experimental Economics Workshop, and the seminar at Xi'an Jiaotong University for helpful suggestions, comments and discussions. Research funded by UC Davis. The datasets and Stata do-file that reproduce the entire analysis reported here are available from http://www.econ.ucdavis.edu/faculty/schipper/.

$\dagger$ Jinhe Center for Economic Research, Xi'an Jiaotong University. Email: liyxjinhe@xjtu.edu.cn

${ }^{\ddagger}$ Department of Economics, University of California, Davis, Email: bcschipper@ucdavis.edu 


\section{Introduction}

Communication is at the heart of strategic interaction. In many economic, political or legal contexts, players have asymmetric information and communication is restricted to verifiable disclosures. Such situations arise for instance between a seller and a buyer, between political parties and voters, in contracting relationships, financial markets, publication of scientific findings etc. They have been studied theoretically in persuasion games starting with seminal work by Grossman (1981), Milgrom (1981), Grossman and Hart (1980), and Milgrom and Roberts (1986) (see Milgrom, 2008, for a review). The central result of this theoretical literature is unraveling of information. Surprisingly, the experimental literature on persuasion games is rather limited (see Section 6 for a discussion). Our work tests experimentally solutions to persuasion games and aims to relate observed data to levels of reasoning.

To gain some intuition about persuasion games, consider a seller and a buyer. The seller has private information about the quality of her good as given by a finite number of quality levels. The buyer's optimal purchase of units of the good depends on the quality. Assume that the higher the quality, the more units he likes to purchase. Before any trade, the seller can provide information to the buyer. This information must be truthful but may be vague. In our setting this means that the seller can disclose a range of quality levels with the provision that the true quality level is contained in this set. In real life this may correspond to a quality certificate. The unraveling argument goes as follows: If the buyer receives the message that the good is of highest quality, then he knows that it has the highest quality because the seller sent a precise message and is not allowed to lie. Thus, a seller who possesses the highest quality good, strictly prefers to disclose it because otherwise she runs the risk of the buyer buying a lower amount. If a buyer receives information that the good is of highest or second highest quality, then he now knows that it is of second highest quality because otherwise the seller would have happily disclosed that it is of highest quality. Thus, a seller having the good of second highest quality, strictly prefers to disclose that it is of highest or second highest quality because otherwise if she mentions also lower quality levels in her message, she runs the risk of the buyer buying less. This argument continues inductively. The punch line is that the seller discloses the true quality (and higher quality levels) and the buyer understands that the good is of lowest quality among the quality levels disclosed by the seller. That is, information unravels.

Originally persuasion games have been solved using sequential equilibrium (e.g., Milgrom and Roberts, 1986). Yet, they can be solved more transparently level-by-level using iterated admissibility and related rationalizability procedures that incorporate some degree of caution or prudence (see Battigalli, 2006, Heifetz, Meier, and Schipper, 2019a). This has the advantage of providing predictions for every finite level of mutual cautious belief in rationality. In our experiments it offers a window to partially observe strategic reasoning of players. Analogous to experimental studies on level- $k$ thinking, it allows us to study the question about levels of reasoning consistent with behavior of sellers and buyers in the experiment. The advantage over the level- $k$ approach is that we do not have to fix level-0 behavior to some more or less arbitrary benchmark. ${ }^{1}$ Surprisingly we find that behavior is consistent with relatively high

\footnotetext{
${ }^{1}$ In fact, it is not really clear to us what the most focal level-0 benchmark should be in persuasion games. The literature on level- $k$ reasoning often assumes uniform randomization at the initial level. Yet, one could argue that in persuasion games naïve honest disclosure may be more natural. With our approach, we can sidestep the
} 
levels of reasoning. The iterative solution concept also allows us to study how the number of quality levels in the persuasion game affects strategic reasoning and unraveling. Essentially it predicts that the number of levels of mutual cautious belief in rationality required for unraveling increases in the number of quality levels.

Our experimental design is as follows: Participants play 30 rounds of persuasion games with random rematching after each round. There are two treatments. In treatment 2-4, participants play the first 15 rounds persuasion games with two quality levels followed by persuasion games with four quality levels in the last 15 rounds. In treatment 4-4, all rounds consist of playing persuasion games with four quality levels. In both treatments, participants are randomly assigned to either the role of the seller or the buyer before each round. After the play, participants complete a Raven's progressive matrices test to evaluate their cognitive abilities. Finally, they complete a questionnaire on demographics.

Our design allows us to address the following questions:

1. What is the empirical distribution of levels of strategic reasoning? Is it relatively low as in previous experimental studies using different games and level- $k$ thinking as the solution concept (e.g. Stahl and Wilson 1995, Nagel, 1995, Costa-Gomes, Crawford, and Broseta 2001, Costa-Gomes and Crawford 2006)? Or is it sufficiently high so as to facilitate unraveling of information in persuasion games? As mentioned earlier, we find relatively high levels of reasoning among participants in our experiment.

2. Is unraveling easier to attain in persuasion games with fewer quality levels than with more quality levels? Consistent with the theory, we find more unraveling in persuasion games with two quality levels than with four quality levels, but the effect remains insignificant.

3. Can participants learn to unravel? We find weak evidence for learning as there are significantly higher levels of reasoning in latter rounds as compared to earlier rounds. Can participants learn better when playing first persuasion games with fewer number of quality levels and only then persuasion games with more quality levels? We reject this hypothesis. In particular, participants seem to have difficulties transferring learning from the persuasion game with two quality levels to the persuasion game with four quality levels.

4. How are levels of strategic reasoning correlated with cognitive ability as for instance evaluated with Raven's progressive matrices test? We find a positive correlation.

Despite the large theoretical body of work on persuasion games, they have surprisingly received only little attention from experimentalists. Exceptions are Forsythe, Isaac, and Palfrey (1989), King and Wallin (1991), Jin, Luca, and Martin (2019a, b), Hagenbach and Perez-Richet (2018), Li and Schipper (2018), Deversi, Ispano, and Schwardmann (2019), and Benndorf, Kübler, and Normann $(2015,2017)$. We defer the detailed discussion and comparison with our experiment to Section 6. Our work is also related to the literature on level- $k$ reasoning. We discuss these connections in Section 6 as well.

The paper is organized as follows: We introduce the theoretical model in the next section. In Section 3, we explain the experimental design. This is followed by Section 4 on hypotheses.

discussion about the most natural initial level. See our discussion in Sections 4 and 6. 
In Section 5 we present the results. Finally, in Section 6 we conclude with a discussion including the related experimental literature. Proofs are relegated to Appendix B. The instruction for the experiment can be found in Appendix C.

\section{Theoretical Model}

\subsection{Experimental Persuasion Game}

Our game is phrased as a game between a seller and a buyer. The seller has a good, which has quality $q \in Q=\{1,2,3,4\}$. Nature moves first and selects quality $q \in Q$. The quality is observed by the seller but not by the buyer. After observing the quality, the seller can provide exactly one message to the buyer. The message specifies a nonempty subset of qualities with the provision that the true quality must be contained in the message. In this sense, the message contains verifiable information (or certified information) although it may be vague. This distinguishes the model also from cheap-talk games. More formally, upon observing quality $q \in Q$, the seller's set of messages is $\mathcal{M}(q):=\left\{M \in 2^{Q} \mid q \in M\right\}$. Since there are four quality levels, for each quality level the seller has exactly eight different messages available. For example, if the true quality is $q=2$, then the set of seller's messages is $\mathcal{M}(2)=\{\{2\},\{1,2\},\{2,3\},\{2,4\},\{1,2,3\},\{1,2,4\},\{2,3,4\},\{1,2,3,4\}\}$.

After receiving the message from the seller, the buyer decides on the quantity he wants to buy. We assume that the quantity is $x \in X:=\{1,2,3,4\}$. The price of each unit is fixed to 4 . Since we abstract from any cost of production or selling, the seller's revenue and profit is $4 \cdot x$. Clearly, the seller prefers the buyer to purchase as many units as possible.

The buyer's payoff depends both on the quality of the good and the quantity purchased. It is given by

$$
12-|x-q|^{\frac{1}{2}}+6 \cdot x^{\frac{2}{3}} \cdot q^{\frac{1}{3}}-4 \cdot x .
$$

We interpret 12 as the buyer's initial wealth. The term $|x-q|^{\frac{1}{2}}$ can be viewed as a penalty of incorrectly guessing the quality value. The term $6 \cdot x^{\frac{2}{3}} \cdot q^{\frac{1}{3}}$ is the value of obtaining $x$ units of the good with quality $q$. Finally, $4 \cdot x$ is the cost of purchasing. The payoff function is set up such that for each possible actual quality $q$, the buyer's payoff is maximized if and only if $x^{*}=q$. For any quantity level purchased different from the optimal level, the buyer is strictly worse off. The marginal payoff from an increase in $x$ to the buyer is increasing in quality $q$ of the good. In particular this means that if the quality is higher, then the buyer's optimal choice of $x$ will also be higher.

We feared that the buyer's payoff function is too complex for experimental participants. That's why we summarize the buyer's payoff function in a simple payoff table that shows for each quality-quantity pair the payoff for the buyer (see Table 1). In fact, our motivation for the payoff function was to be able to present participants in the experiment with such a simple payoff table. Columns refer to quality levels; rows refer to quantities. The number in each cell of the table is rounded to the nearest integer.

In the experiment, we will also consider a version of the persuasion game with just two quality levels in which $Q=\{2,3\}$ with the same set of quantities $X=\{1,2,3,4\}$ and the same 
Table 1: The Buyer's Payoffs in the Experiment

\begin{tabular}{c|c|c|c|c|c|}
\multicolumn{2}{c|}{} & \multicolumn{5}{c}{ Quality } \\
\cline { 3 - 7 } \multicolumn{1}{c|}{} & $q=1$ & $q=2$ & $q=3$ & $q=4$ \\
\cline { 2 - 6 } & $x=1$ & 14 & 10 & 8 & 7 \\
\cline { 2 - 6 } Units & $x=2$ & 8 & 16 & 12 & 11 \\
\cline { 2 - 6 } Purchased & $x=3$ & 4 & 10 & 18 & 14 \\
\cline { 2 - 6 } & $x=4$ & 1 & 7 & 12 & 20 \\
\hline
\end{tabular}

payoff functions.

\subsection{Solution Concept}

It is well known that iterated elimination of strictly dominated strategies is characterized by rationalizability (Pearce, 1984). Similarly, one can characterize iterated elimination of weakly dominated strategies (or iterated admissibility) by prudent rationalizability, in which for each level, players have full support beliefs over the one-step lower level strategies of opponents (i.e., cautious beliefs). ${ }^{2}$ Iterated admissibility is a solution concept for strategic games. Yet, our game is an extensive-form game. Thus, we use the extensive-form analogue to iterated admissibility (or more precisely, the extensive-form prudent rationalizability analogue). It is equivalent to iterated admissibility in the associated normal-form game (see Heifetz, Meier, and Schipper, 2019a, Meier and Schipper, 2012, Shimoji and Watson, 1998, Brandenburger and Friedenberg, 2007).

In extensive-form games, strategies map information sets into actions available at that information sets. For the seller, the quality levels selected by nature represent the information sets. Let a strategy of the seller be $\sigma_{s}: Q \longrightarrow \bigcup_{q \in Q} \mathcal{M}(q)$ such that $\sigma_{s}(q) \in \mathcal{M}(q)$ for all $q \in Q$. That is, if the quality level selected by nature is $q$, the actions/messages available to the seller are $\mathcal{M}(q)$. A seller's strategy assigns to each quality level a subset of quality levels that contains the observed quality level. This captures the verifiable information paradigm in our model. The information sets of the buyer are identified with the messages sent by the seller. Thus, the buyer's strategy is a map $\sigma_{b}: 2^{Q} \backslash\{\emptyset\} \longrightarrow X$. It assigns to each possible message received from the seller a quantity purchased by the buyer. For each player $i \in\{b, s\}$, we denote by $\Sigma_{i}$ player $i$ 's set of strategies.

We say that a move of nature $q \in Q$ and a strategy $\sigma_{s}$ of the seller reaches the information set $Q^{\prime} \in 2^{Q} \backslash\{\emptyset\}$ of the buyer if $\sigma_{s}(q)=Q^{\prime}$.

For any finite set $Y$, denote by $\Delta(Y)$ the set of probability measures on $Y$. Players form beliefs about strategies of the other player and - in the case of the buyer - also moves of nature. A belief system of the seller is a profile of beliefs $\beta_{s}=\left(\beta_{s}(q)\right)_{q \in Q} \in\left(\Delta\left(\Sigma_{b}\right)\right)^{|Q|}$, one for each move of nature. A belief system of the buyer is a profile of $\beta_{b}=\left(\beta_{b}\left(Q^{\prime}\right)\right)_{Q^{\prime} \in 2^{Q} \backslash\{\emptyset\}} \in$ $\prod_{Q^{\prime} \in 2^{Q} \backslash\{\emptyset\}} \Delta\left(Q^{\prime} \times \Sigma_{s}\right)$ such that $\beta_{b}\left(Q^{\prime}\right)$ assign probability 1 to the subset of pairs of moves

\footnotetext{
${ }^{2}$ This argument uses Pearce (1984, Lemma 4).
} 
of nature and strategies of the seller $\left(q, \sigma_{s}\right)$ with $q \in Q^{\prime}$ and $\left(\sigma_{s}\right)^{-1}\left(Q^{\prime}\right) \ni q$. That is, when observing $Q^{\prime}$, the buyer is certain that some move of nature $q$ in $Q^{\prime}$ obtained and the seller chooses a strategy $\sigma_{s}$ that reaches $Q^{\prime}$.

We say that strategy $\sigma_{i}$ of player $i$ is rational with belief system $\beta_{i}$ at her information set if that $\sigma_{i}$ maximizes expected payoffs with respect to the belief prescribed by $\beta_{i}$ at that information set.

Prudent rationalizability is now defined inductively. For each player $i \in\{b, s\}$,

$$
\Sigma_{i}^{0}:=\Sigma_{i}
$$

For each $k \geq 1$,

$$
\begin{aligned}
& B_{s}^{k}:=\left\{\beta_{s}=\left(\beta_{s}(q)\right)_{q \in Q} \mid \text { For every } q \in Q \text { the support of } \beta_{s}(q) \text { is } \Sigma_{b}^{k-1} \cdot\right\} \\
& \Sigma_{s}^{k}:=\left\{\sigma_{s} \in \Sigma_{s}^{k-1} \mid \begin{array}{l}
\text { There exists } \beta_{s}^{k} \in B_{s}^{k} \text { for which } \sigma_{s} \text { is rational } \\
\text { at any move of nature } q \in Q .
\end{array}\right\} \\
& B_{b}^{k}:=\left\{\begin{array}{l|l}
\beta_{b}=\left(\beta_{b}\left(Q^{\prime}\right)\right)_{Q^{\prime} \in 2^{Q} \backslash\{\emptyset\}} & \begin{array}{l}
\text { For every } Q^{\prime} \in 2^{Q} \backslash\{\emptyset\}, \text { if there exists } \\
\text { a move of nature and a seller's strategy } \\
\sigma_{s} \in \Sigma_{s}^{k-1} \text { such that }\left(q, \sigma_{s}\right) \text { reaches } Q^{\prime} \\
\text { then the support of } \beta_{b}\left(Q^{\prime}\right) \text { is the set of } \\
\text { profiles of quality levels and seller's } \\
\text { strategies }\left(q, \sigma_{s}\right) \in\left(Q^{\prime} \times \Sigma_{s}^{k-1}\right) \text { such that } \\
\left(q, \sigma_{s}\right) \text { reach } Q^{\prime} .
\end{array}
\end{array}\right\} \\
& \Sigma_{b}^{k}:=\left\{\sigma_{b} \in \Sigma_{b}^{k-1} \mid \begin{array}{l}
\text { There exists } \beta_{b}^{k} \in B_{b}^{k} \text { for which } \sigma_{b} \text { is rational } \\
\text { at any } Q^{\prime} \in 2^{Q} \backslash\{\emptyset\} .
\end{array}\right\}
\end{aligned}
$$

The set of prudent rationalizable strategies of player $i \in\{b, s\}$ is

$$
\Sigma_{i}^{\infty}:=\bigcap_{k=1}^{\infty} \Sigma_{i}^{k} .
$$

A strategy survives $k$ levels of the prudent rationalizability procedure, if there exists a full support belief on the $k$-level prudent rationalizable strategies of the other player and - in the case of the buyer - feasible moves of nature for which the strategy is rational at every information set of the player. The prudence or cautiousness is captured by full support beliefs. It means that at each level, a player does not completely exclude any of the opponent's remaining strategies and feasible moves of nature. Importantly, in case of the buyer this includes the worst possible quality levels consistent with the message observed. Hence, skepticism about messages comes "for free" through prudence/cautiousness. Heifetz, Meier, and Schipper (2019a) and Meier and Schipper (2012) provide further discussions of the solution concept, including an existence proof for finite extensive-form games and a proof of equivalence to iterated admissibility based on Pearce (1984, Lemma 4).

To see how prudent rationalizability works in persuasion games consider first a persuasion game with two quality levels $Q=\{2,3\}$. At the first level, a buyer receiving a singleton message from the seller knows the true quality (since the seller cannot lie). Thus, he best-responds by 
choosing the quantity equal to the quality level disclosed. At the second level, the seller forms full-support beliefs about first-level prudent rationalizable strategies of the buyer. If her quality is 3 , then she happily tells the buyer because otherwise she runs the risk of the buyer buying less than 3 (since she entertains full-support beliefs). At the third level, the buyer forms fullsupport beliefs over second-level prudent rationalizable strategies of the seller. In particular, if he does not hear the message $\{3\}$ from the seller then he knows that the seller does not have quality 3 since with any second-level prudent rationalizable the seller with quality 3 would have happily disclosed this fact to him. At this point, information unravels in the sense that the buyer deduces complete information about the quality from the seller's message even though some messages may remain vague because a seller with quality 2 sends message $\{2,3\}$. In fact, $\{2,3\}$ is the unique prudent rationalizable action when the seller has quality 2 .

Formally, we say that a strategy profile is unraveling (in terms of information) if

(i) for each quality $q \in Q$, the seller sends message $M \in \mathcal{M}(q)$ with $\min M=q$;

(ii) for each message $M \in 2^{Q} \backslash\{\emptyset\}$, the buyer purchases quantity $x \in X$ with $x=\min M$.

Table 2: Seller's Prudent Rationalizable Strategies with Two Qualities

\begin{tabular}{c||c||c|c|c}
\hline \multicolumn{1}{c||}{ Level of } & \multicolumn{1}{c||}{ Quality selected } & \multicolumn{4}{c}{ Message } \\
reasoning & by nature & $\{2\}$ & $\{3\}$ & $\{2,3\}$ \\
\hline \hline 0 and 1 & $q=2$ & $\sqrt{ }$ & & $\sqrt{ }$ \\
& $q=3$ & & $\sqrt{ }$ & $\sqrt{ }$ \\
\hline 2 and higher & $\begin{array}{c}q=2 \\
q=3\end{array}$ & & & $\sqrt{ }$ \\
& $q$ & $\sqrt{ }$ & \\
\hline
\end{tabular}

Table 3: Buyer's Prudent Rationalizable Strategies with Two Qualities (i.e., Quantity Purchased)

\begin{tabular}{c||c|c|c}
\hline \multicolumn{1}{c||}{ Level of } & \multicolumn{4}{c}{ Message received from seller } \\
reasoning & $\{2\}$ & $\{3\}$ & $\{2,3\}$ \\
\hline \hline 0 & $1,2,3$, or 4 & $1,2,3$, or 4 & $1,2,3$, or 4 \\
\hline 1 and 2 & 2 & 3 & 2 or 3 \\
\hline 3 and higher & 2 & 3 & 2 \\
\hline
\end{tabular}

We characterize prudent rationalizable strategies in our experimental persuasion games as follows:

Proposition 1 The prudent rationalizable strategies of the persuasion game with $Q=\{2,3\}$ are given for the seller and buyer in Tables 2 and 3, respectively. The prudent rationalizable strategies of the persuasion game with $Q=\{1,2,3,4\}$ are given for the seller and buyer in Tables 4 and 5, respectively.

The proof is relegated to Appendix B. Heifetz, Meier, and Schipper (2019a), Schipper and Woo (2019), and Li and Schipper (2018) also apply prudent rationalizability to versions of persuasion games. 

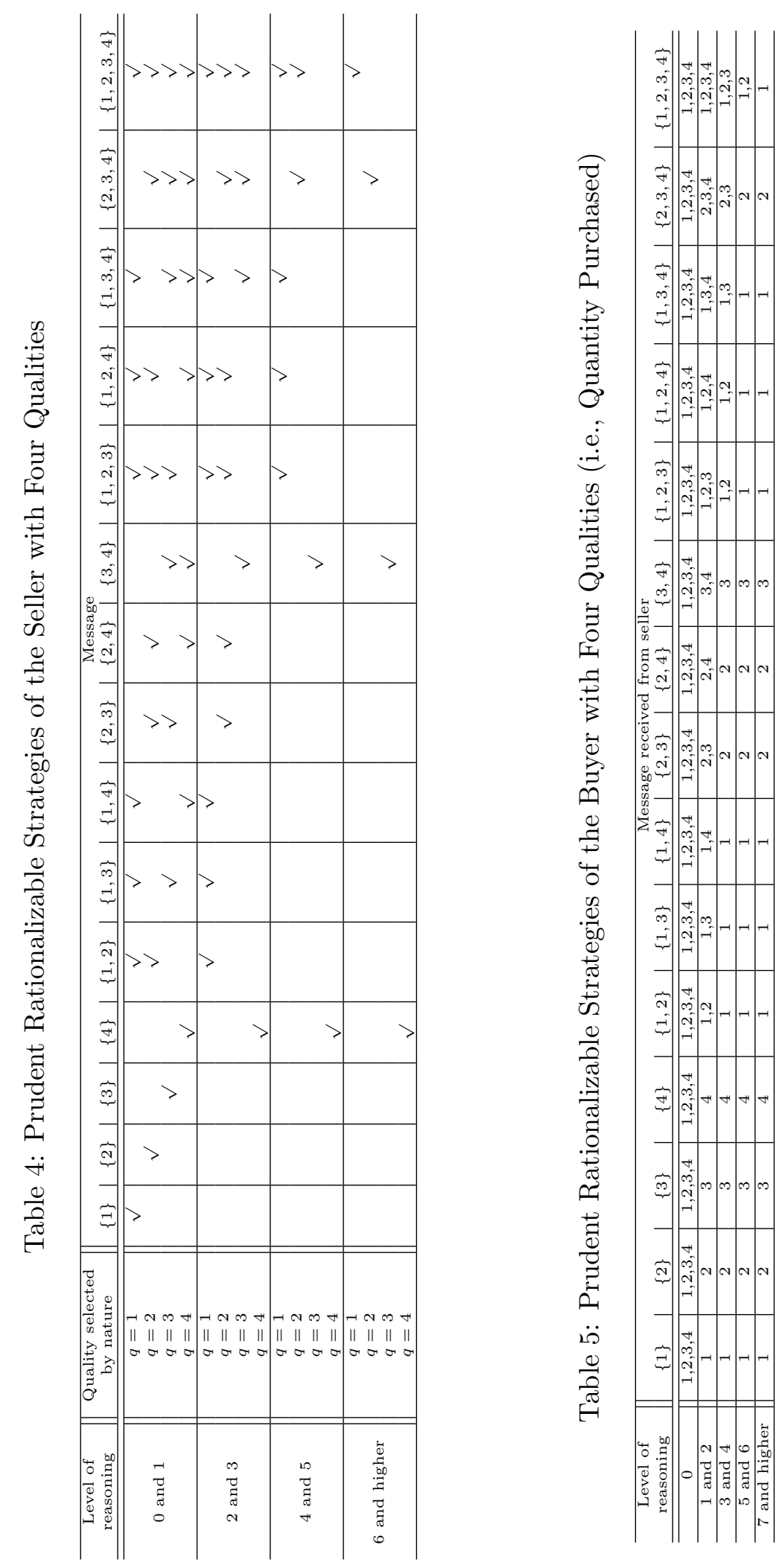
To understand the tables, consider for instance Table 4. The first column indicates the level of reasoning consistent with the various messages that the seller may send. These levels correspond to the levels in the inductive definition of prudent rationalizability (or equivalently, iterated admissibility). Note that for the seller, the solution concept refines strategies only at even levels. That's why there are always two levels of reasoning printed in each cell of the first column. The second column states the move of nature, i.e., the quality of the seller's good selected by nature. The seller's action depends on the move of nature observed by the seller. The next columns state exhaustively all possible messages that the seller may send to the buyer and that are consistent with the quality selected by nature and the levels of reasoning of the seller. The " $\sqrt{ }$ "-sign now indicates which of the seller's messages are consistent with which level of reasoning and quality selected by nature. Similarly, in Table 5 the first column indicates the level of reasoning consistent with the quantity purchased by the buyer. For the buyer, the solution concept refines strategies only at odd levels. The following columns state the quantities consistent with the message received by the buyer and the level of reasoning.

Prudent rationalizability coincides with iterated admissibility or iterated elimination of weakly dominated strategies, a solution concept applied to normal-form games. This follows from results in Shimoji and Watson (1996) and Pearce (1984, Lemma 4); see also Brandenburger and Friedenberg (2007) or Meier and Schipper (2012). That's why in the abstract we referred for simplicity to iterated admissibility.

Prudent rationalizability refines seller's pure sequential equilibrium strategies in persuasion games. For the buyer, it coincides with pure sequential equilibrium strategies. Since sequential equilibrium is standard in persuasion games, we defer the derivation to Appendix A. There we also compare it in more detail with $k$-level prudent rationalizable outcomes.

\section{Experimental Design}

The experiment was programmed in zTree (Fischbacher, 2007) except for a questionnaire on demographics, see Appendix E. ${ }^{3}$ This questionnaire was paper-based and distributed only after the experimental session involving the computerized persuasion games had been completed. Participants were recruited on campus of UC Davis using our ORSEE recruitment system by Greiner (2004).

Upon arrival in the lab, participants received written instructions for the experiment (see Appendix C). They were given sufficient time to read the instructions. After the experimenter went over the written instructions in front of the participants, they were able to ask questions about the instructions and the experiment. These questions were answered by the experimenter in public. The same experimenter conducted all sessions. Note that in the instructions we explained the payoff structure in a straightforward way using a payoff table (see Table 1).

Each session ran one of the following two treatments:

Treatment 2-4: Participants played 15 rounds of a persuasion game with only two

\footnotetext{
${ }^{3}$ During the experiment we decided to add the last two questions to the questionnaire. The data from these last to questions are not used in this paper though. Our intention was to compare to data collected in a companion experiment by Li and Schipper (2018).
} 
quality levels, $\{2,3\}$, followed by 15 rounds of a persuasion game with four quality levels, $\{1,2,3,4\}$.

Treatment 4-4: Participants played all 30 rounds a persuasion game with four quality levels, $\{1,2,3,4\}$.

Each participant was allowed to participate in one session only. Thus, each participant was either assigned to treatment $2-4$ or $4-4$. It is a between-subject experimental design. The treatments differ just in the first 15 rounds. In treatment 2-4, participants play a persuasion game with two quality levels during first 15 rounds, whereas in treatment 4-4, participants play a persuasion game with four qualities throughout. Participants play a persuasion game with four quality levels during the last 15 rounds in both treatments.

At the beginning of each session, participants were randomly assigned into matching-groups of six participants. Each participant stayed in the same matching-group throughout the session. Participants were unaware of matching-groups. At the beginning of each round, each participant was randomly matched to another participant from the same matching-group. The resulting pair of participants played the persuasion game together for one round, one participant being randomly assigned to the role of the seller and the other being the buyer. Participants were randomly rematched within their matching-group after each round. The random rematching should prevent to a large extent repeated games effects over the 30 rounds while allowing us to collect a sufficient number of independent observations. Each matching group is an independent observation. Due to randomly (re-)assigning the role of the buyer or seller to each participant, a participant may find herself in the role of a seller in one round and in the role of a buyer in another. This change of roles should facilitate interactive reasoning. Participants may find it easier to reason about the other player once they have been in a similar role.

In each round, first the seller received information about the quality level selected by nature. The quality level selected by nature was private information of the seller and was not observed by the buyer. Then, the seller decided on what message to send to the buyer (see the seller's screenshot in Appendix D). After receiving the message from the seller, the buyer decided on the quantity to buy (see the buyer's screenshot in Appendix D). Finally, both the buyer and seller were informed about their own payoffs (see the screenshots of payoffs in Appendix D).

After playing persuasion games for 30 rounds, participants completed a Raven's progressive matrices test (Raven et al., 2000) with thirty questions at the computer. Each question consists of a graphic pattern in which one piece is missing. Participants need to select the missing piece out of 8 options in order to complete the pattern. After the Raven's progressive matrices test, participants received the paper-based questionnaire about demographics from the experimenter.

At the end of the session, participants were paid a show-up fee of US $\$ 5.00$ plus earnings from the persuasion games. For each participant, one persuasion game was randomly and independently selected for payment. The payoffs in the persuasion games are already denominated in US dollars. The Raven's progressive matrices test and the questionnaire were not incentivized. This was known to the participants upfront. 


\section{Hypotheses}

Our experimental design allows us to address hypotheses with regard to unraveling, the empirical validity of theoretical solution concepts, levels of reasoning, learning, and the association with cognitive abilities.

As outlined in Section 2, both prudent rationalizability (or equivalently, iterated admissibility) and sequential equilibrium predict unraveling in our persuasion games.

Hypothesis 1 According to theoretical predictions, we expect the frequency of unraveling outcomes to be significantly higher than chance.

To elaborate, we could simply see unraveling by chance. That is, 15 outcomes out of 128 outcomes are unraveling in the experimental persuasion game with four quality levels. ${ }^{4}$ The same holds for the games played in the last 15 rounds of treatment 2-4. In the persuasion game of the first 15 rounds of treatment $2-4$, the chance of unraveling is 3 out of 16 .

We refine Hypothesis 1 with the next hypothesis on the underlying solution concept.

Hypothesis 2 In line with theoretical predictions, we expect participants to play actions consistent with prudent rationalizable strategies.

Since prudent rationalizable strategies refine sequential equilibrium in our persuasion games, the hypothesis also implies that we expect participants to play actions consistent with sequential equilibrium. See Appendix A for a comparative analysis.

Despite our clear theoretical prediction, our prior was nevertheless tilted towards rejecting the hypothesis of playing prudent rationalizable actions and unraveling for serval reasons: First, prudent rationalizability predicts unraveling, there are many solution concepts such as Bayesian Nash equilibrium or extensive-form rationalizability that do not uniquely predict unraveling. These solution concepts do not feature some notion of caution or skepticism. Thus, testing for prudent rationalizability is also a test for cautious beliefs and skepticism. Second, regulators felt a need to mandate disclosure of information in, for instance, financial markets or real estate markets. We suspect that this stems from empirical failure of unraveling in actual persuasion games. Third, the prior literature on level- $k$ reasoning rarely observes more than four levels. Although this literature is based on prior experiments using different games, different samples, and most importantly also a different solution concept with different underlying identifying assumptions, we nevertheless believe it is relevant here if the claim of relatively low levels of reasoning observed in this literature is to be generally valid. One could argue that the guessing game or the travelers' dilemma are even easier to solve than persuasion games as former can be solved with iterated elimination of strictly dominated actions while for persuasion games we need to also assume caution.

At this point, we should discuss assumptions that allow us to partially identify levels of reasoning. In the following analysis, we aim to discern the fractions of participants that can

\footnotetext{
${ }^{4}$ The number of outcomes in the experimental persuasion game is calculated by $4 \times 8 \times 4$, the product of the number of quality levels, the number of messages consistent with each quality level, and the number of quantities available to choose.
} 
reason up to level $k$ for various levels of $k$. Unfortunately, it appears that without additional assumptions the identification of levels of reasoning must be necessarily limited as prudent rationalizability is a reduction procedure on strategies. That is, strategies consistent with higher levels of elimination are also consistent with lower levels of elimination. In order to identify levels of reasoning from behavioral data, we assume the best rationalization principle that is already embodied in prudent rationalizability (and extensive-form rationalizability). That is, our identifying assumption is conceptually consistent with our solution concept. The best rationalization principle stipulates that a player always believes that her opponents are of highest level of reasoning possible that is consistent with the evidence (see Battigalli, 1996). In our identification, we assume that each player in a game is implementing the highest level of reasoning consistent with the evidence we gathered for this player in this game. Of course, there is no reason to go beyond the maximal number of levels required for solving the game. To understand the identifying assumption, consider the case in which the message of a seller given a certain quality level selected by nature is consistent with multiple levels of reasoning and the game is solved after applying $k$-levels of prudent rationalizability. Then, we attribute to the participant the highest level of reasoning $\ell \leq k$ consistent with the message. The identification of levels of reasoning for sellers and buyers is contained in Appendix F. ${ }^{5}$

Besides the best rationalization principle, we use implicitly another identifying assumption that is embodied in the solution concept. Predictions do not only depend on the game but also the context in which players interact, i.e., everything that shapes players' beliefs about each others' rationality and play, beliefs about that etc. ${ }^{6}$ It is true that a version $k$-level of mutual cautious belief in rationality characterizes $k$-level iterated admissible strategies (and thus, $k$-level prudent rationalizable strategies) if the type space is "rich". It is not true though when the type space is not "rich" but restricted to a particular context (e.g., Brandenburger, Friedenberg, an Keisler, 2008, Theorems 8.1 and 9.1). Such a restriction could come in form of first-level beliefs or equivalently assumptions on the behavior of "level-0" players as in the level$k$ literature. Our solution concept does not impose such restrictions here. Instead we assume implicitly that players are able to entertain the thought that opponents can be of any type consistent with $k$-level mutual cautious belief in rationality. The implicit assumption of a rich type space cannot be tested in our data. ${ }^{7}$ We will use these assumptions to partially identify levels of reasoning especially in our descriptive analysis of levels. In Section 6 we provide further discussion of identifying assumptions when we compare our solution concept to the literature

\footnotetext{
${ }^{5}$ Levels of reasoning could be fully identified in a clever-designed game (see for instance, Kneeland 2015). Yet, we are interested in levels of reasoning in persuasion games. It is not the case that empirically observed levels of reasoning are necessarily stable across different games (e.g., Georganas, Healy, and Weber, 2015).

${ }^{6}$ This is a general problem for testing solution concepts in games that is rarely mentioned in the literature; for an exception see Brandenburger, Danieli, and Friedenberg (2019).

${ }^{7}$ The assumption of a "rich" type space comes with a couple of caveats: There is a debate about what it means to have a "rich" type space for characterizing iterated admissibility. There are various proposals in the literature (Brandenburger, Friedenberg, Keisler, 2008, Lee and Keisler, 2015, Lee, 2016, Dekel, Friedenberg, Siniscalchi, 2016, Yang, 2016, Catonini and De Vito, 2019, 2018, Heifetz, Meier, and Schipper, 2019b etc.). Some differ with respect to continuity assumptions that may be interpreted as extreme degrees of caution w.r.t. to types of other players. We do not know how to test such assumptions in experiments. Second, we use prudent rationalizability, an extensive-form version of iterated admissibility. Although this solution concept is level-bylevel strategy-equivalent to iterated admissibility applied to the associated normal-form, it is conceptually more appropriate to characterize it in "rich" type space for dynamic cautious beliefs, something we have not seen in the literature yet.
} 
on level- $k$ reasoning.

In our persuasion game with four quality levels, if nature chooses qualities 1 or 2 , then at least six levels of reasoning are required from the seller for excluding play of an action inconsistent with prudent rationalizability. Similarly, if nature chooses quality 3 , then at least four levels of reasoning are required from the seller for excluding any action inconsistent with prudent rationalizability. And if nature chooses quality 4, then just two levels are required (see Table 4). Thus, with regard to the seller's behavior, we expect that the prevalence of actions consistent with prudent rationalizable strategies is increasing in the quality chosen by nature. The hypothesis applies primarily to the persuasion game with four quality levels. The persuasion game with two quality levels is not rich enough to derive such comparative statics.

A similar hypothesis can be developed with regard to the buyer's behavior. For any singleton message received from the seller, the buyer just needs at least one level of reasoning for choosing a quantity consistent with prudent rationalizability. For any message containing two elements, the buyer needs at least three levels of reasoning for choosing a quantity consistent with prudent rationalizability. For any message containing three elements, the buyer needs at least five levels. Finally for the message containing all four qualities, the buyer needs at least seven levels of reasoning. An analogous hypothesis applies to the buyer in the persuasion game with two quality levels. We summarize the discussion as follows:

Hypothesis 3 In line with theoretical predictions for the seller, we expect that in persuasion games with four quality levels the frequency of actions consistent with prudent rationalizable strategies is increasing in the quality chosen by nature.

For the buyer, we expect that the frequency of demanded quantities consistent with prudent rationalizable strategies is decreasing in the size of the message received from the seller.

Differences between behavior of the first 15 rounds of treatments 2-4 and 4-4 can also provide evidence for the relevance of levels of reasoning. According to theoretical predictions based on prudent rationalizability, having a lower number of qualities in the persuasion game of treatment 2-4 requires less levels of reasoning for unraveling to occur than in the persuasion game with a higher number of qualities in treatment 4-4. This motivates the following hypothesis.

Hypothesis 4 In line with theoretical predictions, we expect that the frequency of unraveling is significantly higher in the first 15 rounds of treatment 2-4 than in the first 15 rounds of treatment $4-4$.

Although participants may not display too much unraveling in the first few rounds of the experiment, they may be able to learn over time. Since our experiment lasts for 30 rounds, we should be able to observe some learning. While in our experiment we cannot disentangle learning of higher levels of reasoning from learning about the context of play (e.g., restrictions of first-order beliefs about opponent's play), it allows us to generally conclude whether or not players are learning to play more sophisticated as captured by changes in the frequency of unraveling and fitted distribution of levels of reasoning. 
Hypothesis $\mathbf{5}$ We expect to see evidence for learning to unravel. More precisely, in treatment 4-4 we expect that average unraveling is significantly larger in the last 15 rounds than in the first 15 rounds.

Hypothesis 6 In treatment 4-4, the cumulative distribution of levels of reasoning in the last 15 rounds first-order stochastically dominates the cumulative distribution of levels of reasoning in the first 15 rounds.

In treatment 2-4, participants play first 15 rounds a persuasion game with two qualities followed by 15 rounds of a persuasion game with four quality levels. Our intuition suggests to us that playing first a simpler persuasion game should facilitate playing in a more complex persuasion game. The inductive nature of unraveling argument may be easier to grasp in the simpler setting and then easily generalized to the game with more quality levels. More generally, we usually learn by starting out with simpler problems and progressing to more complicated problems. There is weak evidence for transfer of learning from a simpler game to a more complex game by Duwfenberg, Sundaram, and Butler (2010) although they study a class of games very different from ours. ${ }^{8}$ This motivates the following hypotheses on transfer of learning:

Hypothesis 7 We expect average unraveling in the last 15 rounds of treatment 2-4 to be significantly higher than average unraveling in the last 15 rounds of treatment 4-4.

Hypothesis 8 The cumulative distribution over levels of reasoning in the last 15 rounds of treatment 2-4 first-order stochastically dominates the cumulative distribution over levels of reasoning in the last 15 rounds in treatment 4-4.

Finally, our design allows us to explore the association between levels of reasoning in persuasion games and cognitive abilities as measured with the Raven's progressive matrices test. Among the many facets of cognitive ability, Raven's progressive matrices test is said to focus on "meaning making" ability or "eductive ability", i.e., the ability to draw meaningful inferences from rudimentary confusing experience (Raven, 2008). Such an ability may be useful in many contexts including strategic interaction. An association between scores from Raven's progressive matrices test and level- $k$ reasoning has already been suggested in the literature. Gill and Prowse (2016) find that participants with higher scores in the Raven's test are more likely to choose equilibrium actions in the $p$-beauty contest, and they also converge more frequently to equilibrium play and earn more even as behavior approaches the equilibrium prediction.

Hypothesis 9 The level of reasoning is significantly positively correlated with the score on the Raven's progressive matrices test.

\footnotetext{
${ }^{8}$ See also Cooper and Kagel $(2008,2009)$ for somewhat mixed experimental results on transfer of learning across games.
} 
Table 6: Demographics of Our Sample

\begin{tabular}{l||r|r|r}
\hline Variable & Number & Mean & Std. Dev. \\
\hline \hline Participants & 372 & & \\
Female & 215 & 0.58 & \\
Age & 371 & 20.98 & 2.31 \\
\hline White & 84 & 0.23 & \\
Asian & 234 & 0.63 & \\
Black or African & 3 & 0.01 & \\
Mixed or Others & 51 & 0.13 & \\
\hline GPA & 366 & 3.19 & 0.48 \\
Math & 5 & 0.01 & \\
All Sciences & 212 & 0.60 & \\
Engineering & 33 & 0.09 & \\
Economics & 79 & 0.21 & \\
Other Social Sciences & 87 & 0.23 & \\
Humanities and Arts & 23 & 0.06 & \\
\hline
\end{tabular}

Note: The major classification is taken from majors by college from https://www.ucdavis.edu/majors/college. The sum of the mean of majors is greater than 1 since we take double-major into account.

\section{Results}

The datasets and Stata do-file that reproduce the entire analysis reported here are available from http://www. econ.ucdavis.edu/faculty/schipper/.

The experiment was conducted in a computer lab of University of California, Davis during spring quarter 2016. There were 20 experimental sessions; 10 sessions for each treatment. A total of 372 participants joined the experiment; 186 participants in each treatment. Table 6 summarizes demographic information of our sample. The average payment including the showup fee was $\$ 17.48$ with a maximum of $\$ 25$ and a minimum of $\$ 6$. Each experimental session lasted for about 1 hour and 15 minutes.

\section{$5.1 \quad$ Unraveling}

We observe a high frequency of unraveling outcomes in both treatments. In treatment 4-4, average unraveling over all 30 rounds is $75.70 \%$ while expected unraveling by random choice would be $11.7 \%$. Former is significantly higher than latter $(p$-value $<0.001)$ using $\chi^{2}$-goodness of fit test clustered on matching groups. In treatment 2-4 average unraveling over the first 15 rounds (when persuasion games with two quality levels are played) is $73.05 \%$ while expected unraveling by random choice would be $18.75 \%$. Again, former is significantly higher than latter ( $p$-value $<0.001, \chi^{2}$-goodness of fit clustered on matching groups). In the last 15 rounds of treatment $2-4$, average unraveling over 15 rounds is $68.89 \%$. Although this is lower than in the first 15 rounds, the drop is probably due to playing persuasion games with two quality levels in the first 15 rounds. In any case, this is significantly higher than unraveling by random choice ( $p$-value $<0.001, \chi^{2}$-goodness of fit clustered on matching groups). 
Observation 1 The frequency of unraveling outcomes is significantly higher than chance. We cannot reject Hypothesis 1.

\subsection{Empirical Validity of Solution Concept}

How descriptive is prudent rationalizability for behavior in persuasion games? Figure 1 shows histograms of messages sent by sellers. We print separate histograms for each quality selected by nature. Levels of reasoning are indicated with the shade of the bars in the histograms. The darker the shade of a message, the higher the level of reasoning consistent with it. Clearly, for each quality, the prudent rationalizable action is supported by the highest frequency among all actions. Between $88 \%$ to $35 \%$ of empirical actions can be rationalized by prudent rationalizability depending on the quality selected by nature. We conclude that the solution concept rationalizes a substantial fraction of sellers' actions.

A similar picture emerges with respect to buyers' behavior. Figure 2 presents histograms of buyers' quantity choices. For each message sent by the seller, there is a histogram of quantity choices. Again, darker shades of the bar indicate higher levels of reasoning. For each message sent by the seller, the highest bar corresponds to the prudent rationalizable quantity choice of the buyer. Between $99 \%$ and $59 \%$ of buyer's quantity choices can be rationalized by prudent rationalizability. Thus, a substantial fraction of buyers' actions can be rationalized by the solution concept.

Observation 2 Prudent rationalizability describes a substantial fraction of behavior. Depending on player role and information set, prudent rationalizability rationalizes between 99\% and $35 \%$ of actions in the experiment. We cannot reject Hypothesis 2.

\subsection{Levels of Reasoning}

In Figure 1 we observe that the frequencies of prudent rationalizable messages of sellers are increasing in quality levels, which we believe is due to the fact that the lower the quality selected by nature the more levels of reasoning are required for prudent rationalizable actions.

Similarly, in Figure 2 we note that the frequency of prudent rationalizable quantities of the buyer is decreasing in the size of the message received from the seller, which again we believe is due to the fact that more levels of reasoning are required for the prudent rationalizable quantities when the size of seller's message is larger.

Observation 3 In persuasion games with four quality levels the frequency of sellers' actions consistent with prudent rationalizable strategies is increasing in the quality chosen by nature. Moreover, the frequency of buyers' actions consistent with prudent rationalizable strategies is decreasing in the size of the message received from the seller. We cannot reject Hypotheses 3.

The preceding analysis suggests already that if participants were classified by levels, we should see a distribution of levels that puts relatively large mass on higher levels. Given the 
Figure 1: Histograms of Messages sent by Sellers
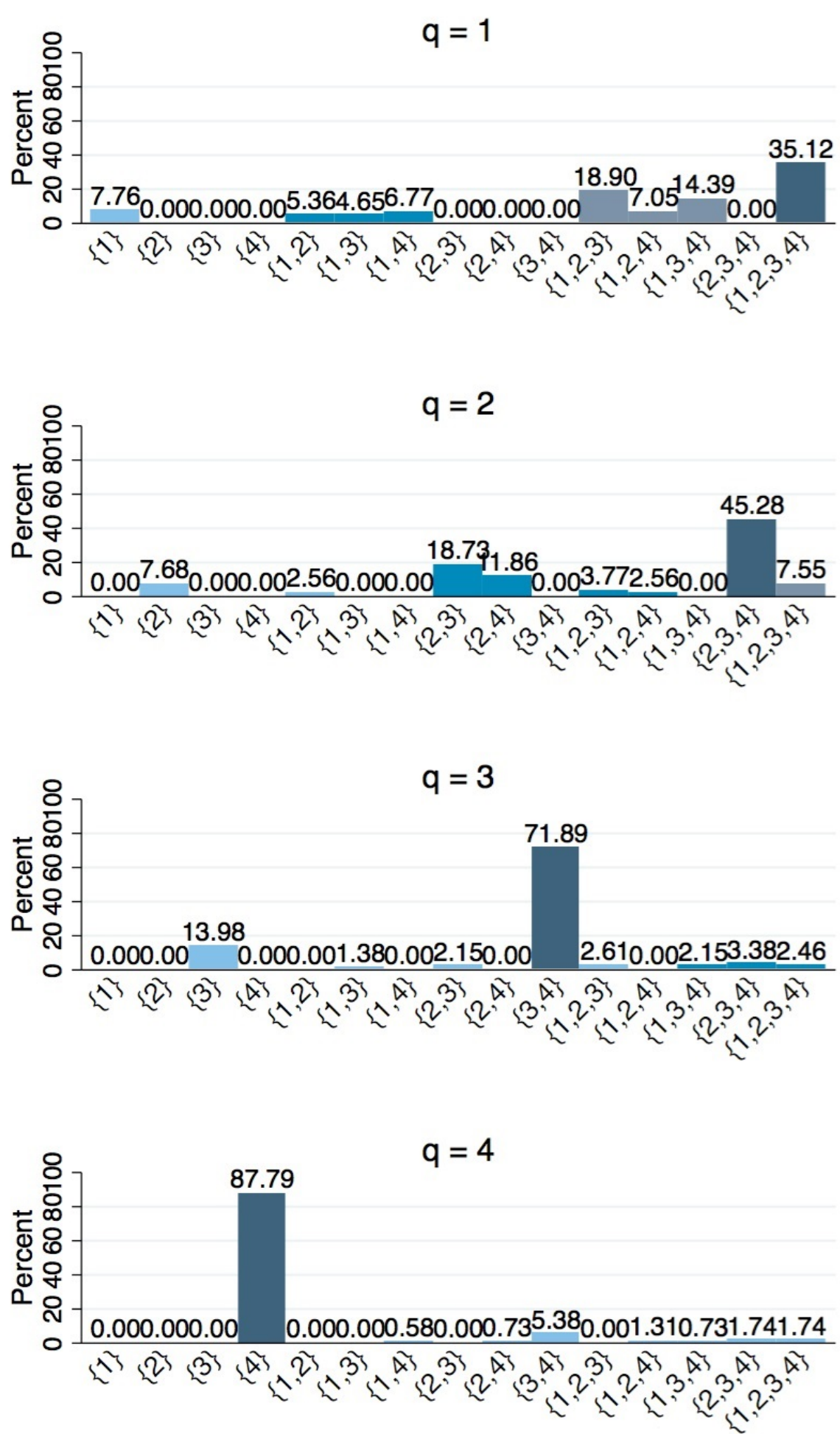

Darker shades indicate higher levels of reasoning. 
Figure 2: Histograms of Quantities chosen by Buyers
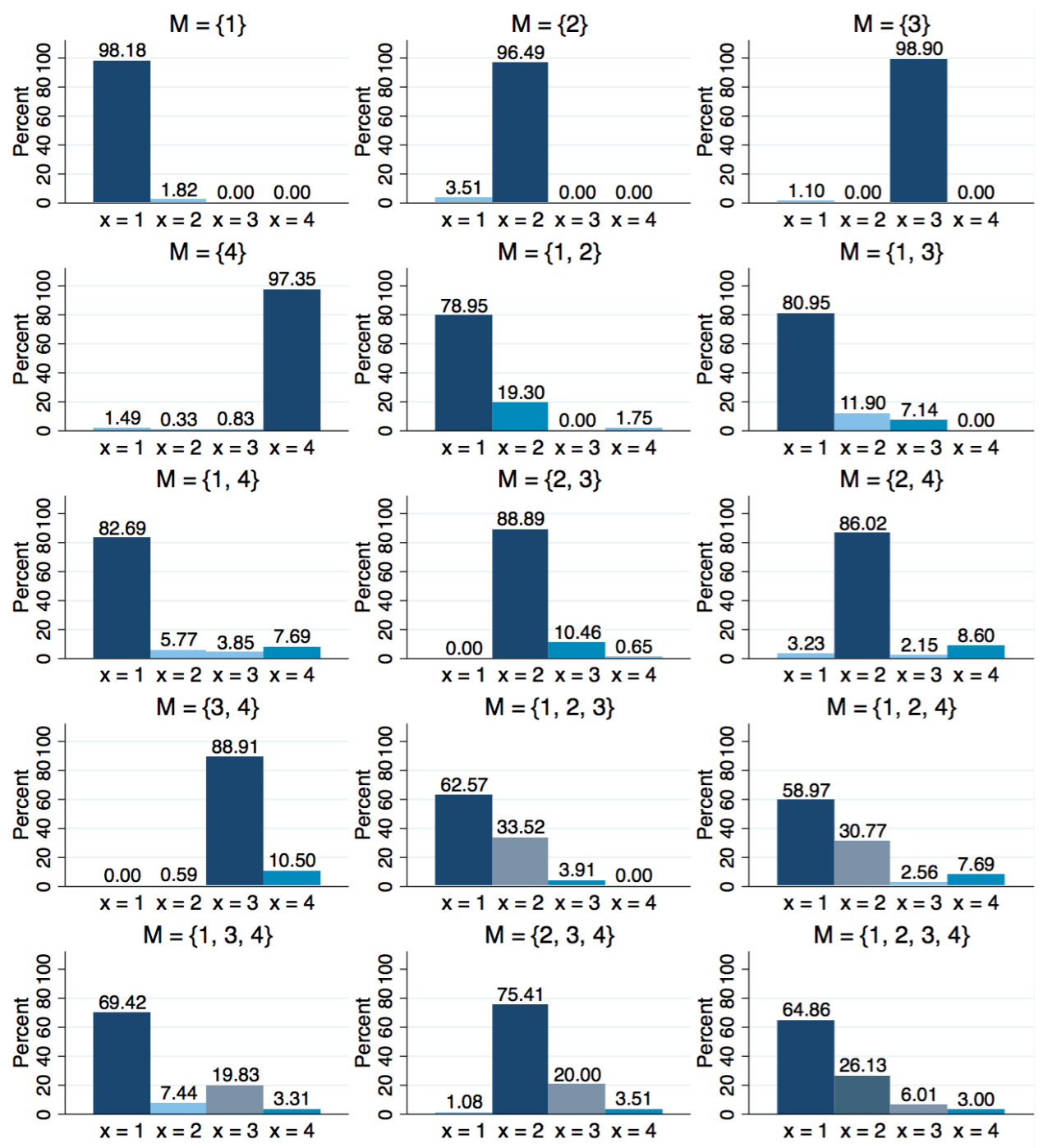
Figure 3: Sellers' Distribution of Levels by Round in Treatment 4-4

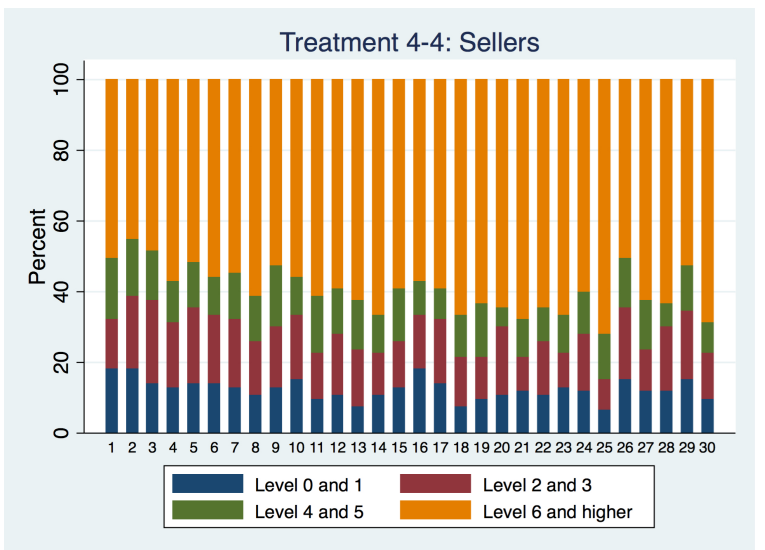

Figure 4: Buyers' Distribution of Levels by Round in Treatment 4-4

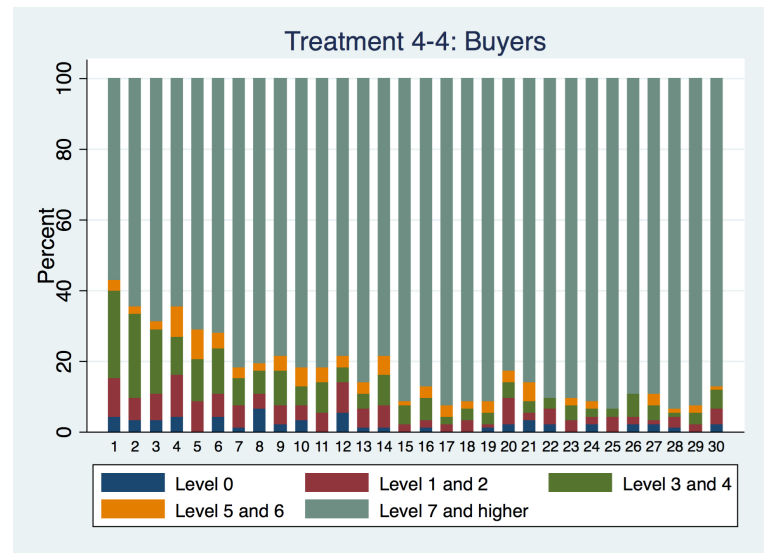

partial identification of levels in our data (as discussed in Section 4), there are different ways of classifying participants into levels of reasoning though.

First, consider a classification that allows the level of reasoning attributed to a participant to vary with her role and the round of play. For instance, a participant may be level 2 as a seller but level 5 as a buyer. Allowing the classification to depend on the role is justified by the fact that the problem to solve in the role of the buyer differs qualitatively from the role of the seller as latter is the leader while former is the follower. Moreover, prudent rationalizability refines strategies only at even levels for the seller and only at odd levels for the buyer. Allowing the classification to depend on the round of play treats each game separately. It is justified when we consider that players may learn to reason or when the context of each instance of the game is shaped by the experiences in prior games. Although we cannot measure this context, i.e., it is hard to elicit the initial beliefs of each player at the beginning of each game not just about the opponent's play but also higher order beliefs, we still find it meaningful to summarize data in each round by fitted levels of reasoning.

Figures 3 and 4 show the distribution of levels of sellers and buyers, respectively, in each round of treatment $4-4$. We see that over $50 \%$ of the sellers are consistent with the highest level of reasoning in treatment 4-4. This number is even higher for buyers where depending on the round roughly 60 to $90 \%$ of the buyers are consistent with the highest level of reasoning.

For treatment 2-4, Figures 5 and 6 show a similar picture. In the first 15 rounds, where less levels of reasoning are required to solve the game with two quality levels, roughly 70 to $80 \%$ of the sellers are consistent with the highest level of reasoning. It drops to roughly $60 \%$ in the last 15 rounds when participants play games with four quality levels. This is still substantial and comparable with the level of reasoning seen in treatment 4-4. For buyers, 70 to $90 \%$ are consistent with the highest level of reasoning in the first 15 rounds and roughly 55 to $90 \%$ in the last 15 rounds. Again, latter numbers are consistent with what we see for buyers in treatment $4-4$.

So far, the analysis treated levels of reasoning as role and game specific. An alternative view is to treat levels as a feature of the participant and its role only without allowing it to 
Figure 5: Sellers' Distribution of Levels by Round in Treatment 2-4

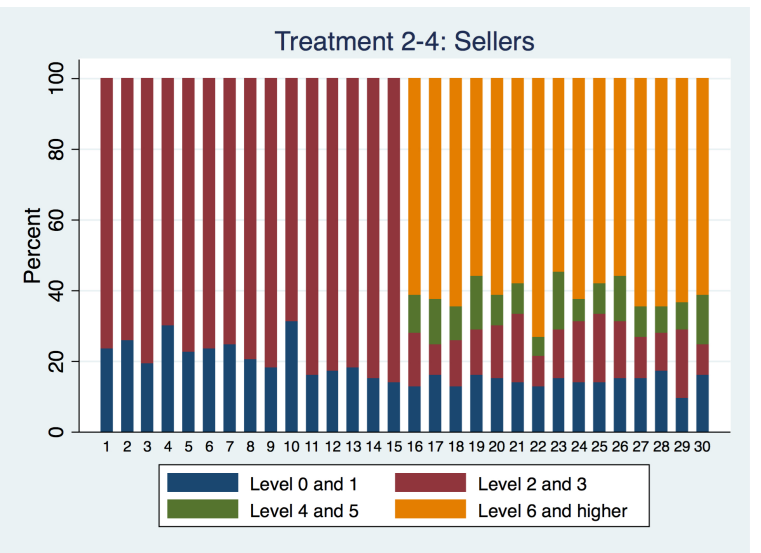

Figure 6: Buyers' Distribution of Levels by Round in Treatment 2-4

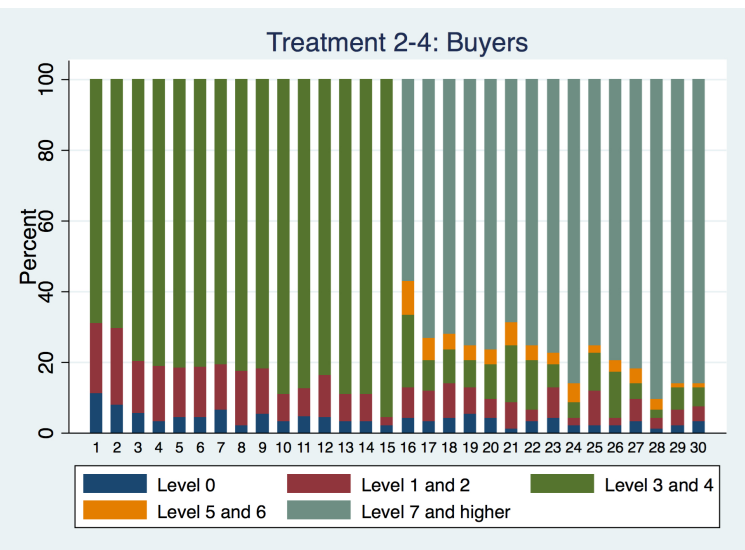

depend on the game. ${ }^{9}$ According to this view, if a participant displays behavior consistent with level 6 in one game but behavior only consistent with level 2 in another, then he cannot be classified as a consistent level 6 reasoner. Given the complex human nature, it may be heroic to assume that every participant in our experiment is consistent with a certain level of reasoning in every round. ${ }^{10}$ Therefore we classify a participant as level $k$ if level $k$ is the highest level (s)he is consistent with at least $86 \frac{2}{3} \%$ of the times. ${ }^{11}$ One may argue that allowing for mistakes is somewhat conceptually inconsistent with prudent rationalizability or extensive-form rationalizability as in these solution concepts a player does not discard unexpected behavior of an opponent as a plain mistake but rather tries to attribute as much rationality to the opponent as possible. ${ }^{12}$ Yet, from a statistical perspective this view looks too orthodox. In any case, the participant-role specific identification of levels is certainly more conservative than the earlier participant-round-role specific classification.

Using such a participant-role specific classification over all rounds, we characterize $14.52 \%$ of the sellers as having level 6 or higher and $61.83 \%$ of the buyers are having level 7 or higher in treatment 4-4 with the persuasion game with four quality levels. Figures 7 and 8 show cumulative distributions of levels of all rounds in treatment 4-4 for sellers and buyers, respectively. Note that the levels on the horizontal axis are in reverse order, starting with the highest relevant level. Clearly, it is certainly not the case that reasoning of level 4 or higher is almost non-existent as often reported in the literature on level- $k$ reasoning in different games.

A third alternative is to focus just on the first round of play when classifying participants by levels. The reason is that the solution concept does not incorporate beliefs over play shaped

\footnotetext{
${ }^{9}$ Both views may be justified. Georganas, Healy, and Weber (2015) find no correlation of levels of reasoning between games of different families. Yet, they find fairly consistent levels of reasoning within a each family of games.

${ }^{10}$ Typically, the level- $k$ literature features maximum likelihood estimations. Such estimations allow for some mistakes.

${ }^{11}$ This means that the participant can be inconsistent at most 4 times over 30 rounds. Since we want to analyze the first and last 15 rounds separately, we need to allow for an even number of inconsistencies. This explains our threshold $86 \frac{2}{3} \%$ rather than $90 \%$.

${ }^{12}$ This is also the main reason for why we don't attempt a maximum likelihood estimation.
} 
Figure 7: Sellers' Distribution of Levels Over All Rounds in Treatment 4-4

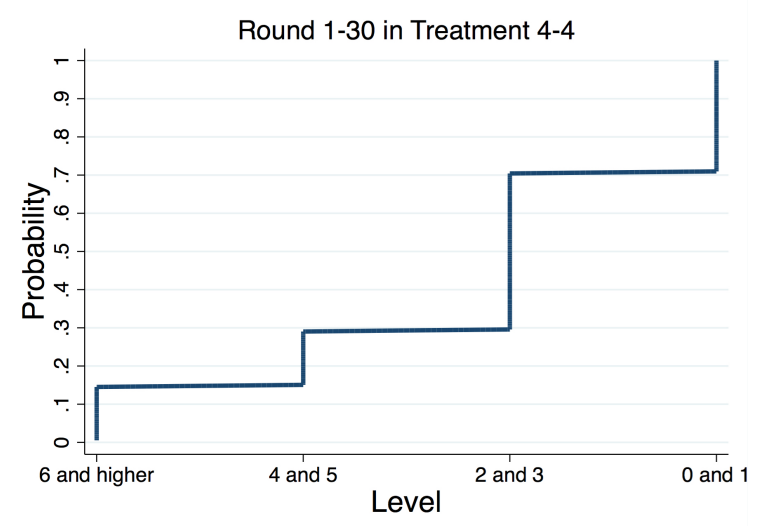

Figure 8: Buyers' Distribution of Levels Over All Rounds in Treatment 4-4

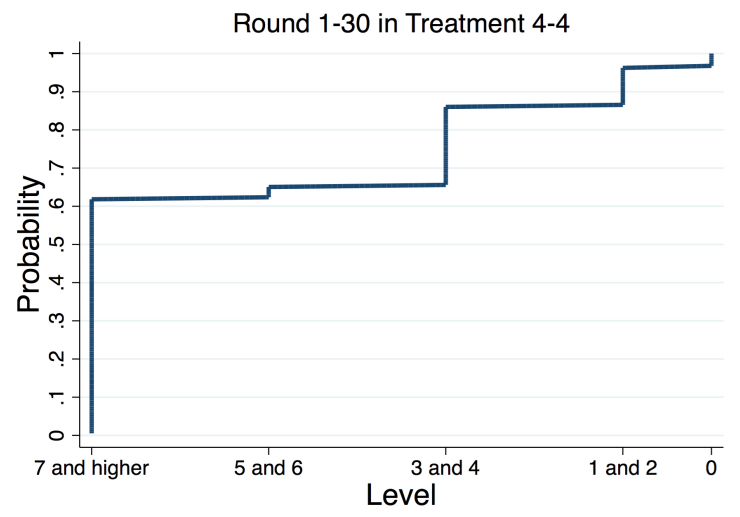

by interaction and learning in prior games. Instead they are just shaped by a version of $k$-level mutual cautious beliefs in rationality in a rich type space. With this view, it is not necessarily meaningful to compare levels of reasoning across rounds as learning about play can shape behavior even though actual levels of reasoning may not change.

While the distribution of levels in the first round are also visualized in prior figures, we tabulated them in Table 7 for better readability. In treatment $4-4$, about $50 \%$ of the sellers and more than $55 \%$ of the buyers can be classified with levels 6 and 7 , respectively, already in the first round. In treatment 2-4, more than $75 \%$ of the sellers and more than $68 \%$ of the buyers can be classified with levels 2 and 3, respectively, in the first round. These are the highest levels of prudent rationalizability at which strategies are refined by the solution concept and solve the game.

Table 7: Distribution of Levels in the First Round

\begin{tabular}{l|c||l|l||l|l||l|r}
\hline \multicolumn{3}{c||}{ Sellers } & \multicolumn{3}{c}{ Buyers } \\
\multicolumn{2}{c|}{ Treatment 2-4 } & \multicolumn{2}{c||}{ Treatment 4-4 } & \multicolumn{2}{c}{ Treatment 2-4 } & \multicolumn{2}{c}{ Treatment 4-4 } \\
\hline Levels 0-1 & $23.66 \%$ & Levels 0-1 & $18.28 \%$ & Level 0 & $11.11 \%$ & Level 0 & $4.30 \%$ \\
Levels 2-... & $76.34 \%$ & Levels 2-3 & $13.98 \%$ & Levels 1-2 & $20.00 \%$ & Levels 1-2 & $10.75 \%$ \\
& & Levels 4-5 & $17.20 \%$ & Levels 3-... & $68.89 \%$ & Levels 3-4 & $24.73 \%$ \\
& & Levels 6-... & $50.54 \%$ & & & Levels 5-6 & $3.23 \%$ \\
& & & & & & Levels 7-... & $56.99 \%$ \\
\hline
\end{tabular}

Other features of our experimental design can also be used to evaluate the relevance of levels of reasoning. For instance, comparison of the behavior in the first 15 rounds of treatment 2-4 with behavior in first 15 rounds of treatment 4-4 may offers a glimpse on the relevance of levels of reasoning. This is because the persuasion game with four qualities levels in treatment 4-4 requires more levels of reasoning for unraveling than the game with two quality levels in treatment $2-4$.

Table 9 shows sample means of unraveling in the first 15 rounds for both treatments. The persuasion games with two quality levels show more frequent unraveling than the persuasion 
games with four quality levels. The difference is insignificant though $(p$-value $=0.146)$. The variation of unraveling frequencies across rounds also resembles the same pattern. In Figure 9, the whole series of unraveling outcomes in the first 15 rounds of treatment 2-4 lie right above the first 15 rounds of treatment 4-4, but the vertical distance is just about $5 \%$ at most.

The ability for unraveling may be correlated with demographic variables such as GPA or major of studies. Although we do not have precise theory about how exactly these covariates affect unraveling, we can use these variables as controls. To this end, we further analyze the difference between the two treatments using both probit and logit regressions (since unraveling is a binary variable). We regress unraveling on a dummy for treatment 2-4, a dummy for rounds 16-30, the interaction of those two, and controls for demographic variables. The results are presented in Table 8.

Table 8: Binary Regression Results of Unraveling

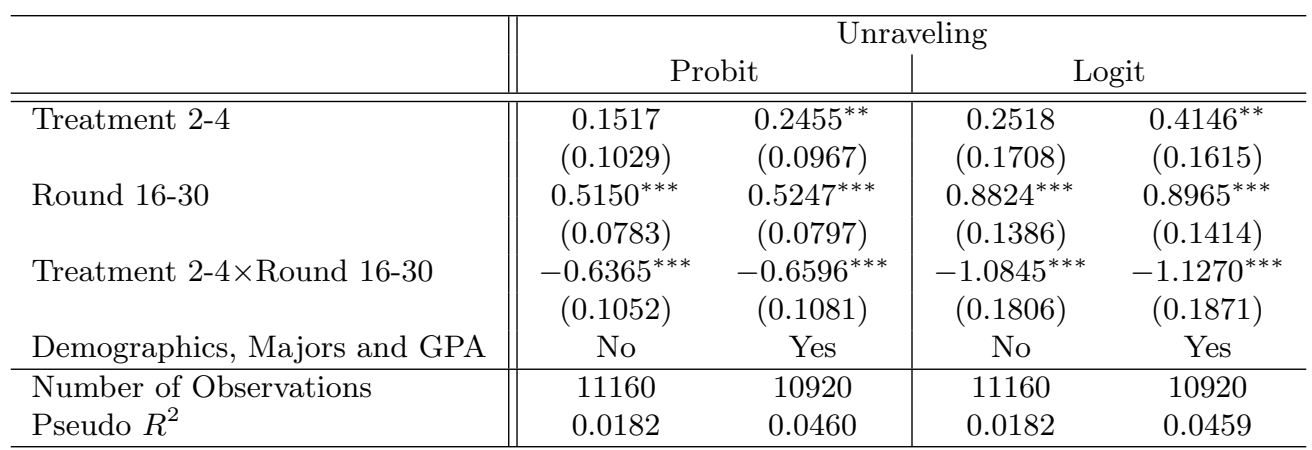

Note: We use robust standard errors clustered at group level. Significance levels: *10\%, **5\%, and $* * * 1 \%$.

The coefficient of treatment 2-4 quantifies the treatment effect for the first 15 rounds between the two treatments. When controlling for demographics in the probit specification, the coefficient, 0.2455 , implies that playing the persuasion games with two quality levels increases the probability of unraveling by 0.077 , which is significant at $5 \%$. However the increase in probability, 0.077 , is close to the difference of random unraveling by chance between the two treatments, which is $\frac{3}{16}-\frac{15}{128}=0.0703$. The $t$-test on the marginal effect of treatment $2-4$ being larger than 0.0703 shows a $t$-statistic of $0.2174(p$-value $=0.414)$. Therefore, we cannot conclude that the positive difference of unraveling in the first 15 rounds between treatment 2-4 and treatment 4-4 is significantly higher than what is implied by chance.

The logit regression confirms our findings of the probit regression. When controlling for demographics, the coefficient estimate for treatment 2-4 is 0.4146 , which is statistically significant at 5\%. It means that the odds ratio of attaining unraveling in the first 15 rounds of treatment $2-4$ is $\exp (0.4146)=1.5138$. If the outcomes are randomly chosen, the odds of attaining unraveling in the first 15 rounds of treatment $2-4$ are $\frac{3}{13}$ and the odds of attaining unraveling in the first 15 rounds of 4- 4 treatment are $\frac{15}{113}$, and that leads to the odds-ratio of attaining unraveling by chance between two treatments of $\frac{3}{13} / \frac{15}{113}=1.7385$, which is larger than 1.5138 . Again, the results from the logit regressions reject Hypothesis 4.

Observation 4 Although the frequency of unraveling is significantly higher in the first 15 
rounds of treatment 2-4 than in the first 15 rounds of treatment 4-4, the difference is not significantly different from the difference of random unraveling in both treatments. We reject Hypothesis 4 .

The observation is not to say that levels of reasoning are irrelevant for outcomes. Rather, simpler games may also induce participants to exert less reasoning efforts and thus not more unraveling as compared to more complex games. ${ }^{13}$ Finally, note that Observation 4 is not an artefact of a "ceiling effect". The unraveling frequencies are sufficiently bounded away from one so that they could have been significantly different from each other.

\subsection{Learning}

Table 9 shows the average unraveling in percentage for both treatments separated by the first 15 rounds and the last 15 rounds. In treatment $4-4$, there is significantly more unraveling in the last 15 rounds than in the first 15 rounds $(p$-value $<0.001)$. This suggests some learning to unravel in treatment $4-4$.

Table 9: Average Unraveling

\begin{tabular}{c||cc|c}
\hline Round & Treatment 4-4 & Treatment 2-4 & Difference \\
\hline \hline $1-15$ & 67.81 & 73.05 & -5.24 \\
& & & $(3.55)$ \\
$16-30$ & 83.58 & 68.89 & $14.69^{* * *}$ \\
& & & $(4.23)$ \\
\hline \hline Difference & $-15.77^{* * *}$ & 4.16 & \\
& $(2.31)$ & $(2.51)$ & \\
\hline
\end{tabular}

Note: We use robust standard errors clustered at group level. Significance levels: ${ }^{*} 10 \%, * * 5 \%$, and $* * * 1 \%$.

In Figure 9, we show round-by-round frequency of unraveling (over all pairs of participants) for both treatments. We also fit trend lines separately for the first and last 15 rounds of each treatment. Again, the figure suggests that with respect to treatment $4-4$ there is learning. Moreover, typically for a learning curve, it is steeper in earlier rounds than in latter rounds.

Observation 5 In treatment 4-4, average unraveling is significantly larger in the last 15 rounds than in the first 15 rounds. This is evidence for learning of unraveling. We cannot reject Hypothesis 5.

Participants may become more comfortable with playing the game over time. This learning can be summarized by the dynamics of distributions of levels of reasoning even though it should just be interpreted as an "as if" analysis as learning may also occur w.r.t. the context of each game (e.g., restrictions on first-level beliefs about opponent's play) without changes in the actual levels. We can see already in Figures 3 to 6 that the fraction of participants with behavior consistent with higher and higher levels of reasoning is increasing at least for

\footnotetext{
${ }^{13}$ See Alaoui and Penta (2017) for an approach to level- $k$ reasoning in which the effort of reasoning is endogenized.
} 
Figure 9: Frequency of Unraveling in Round 1 - 15 vs. Round 16 - 30 by Treatment

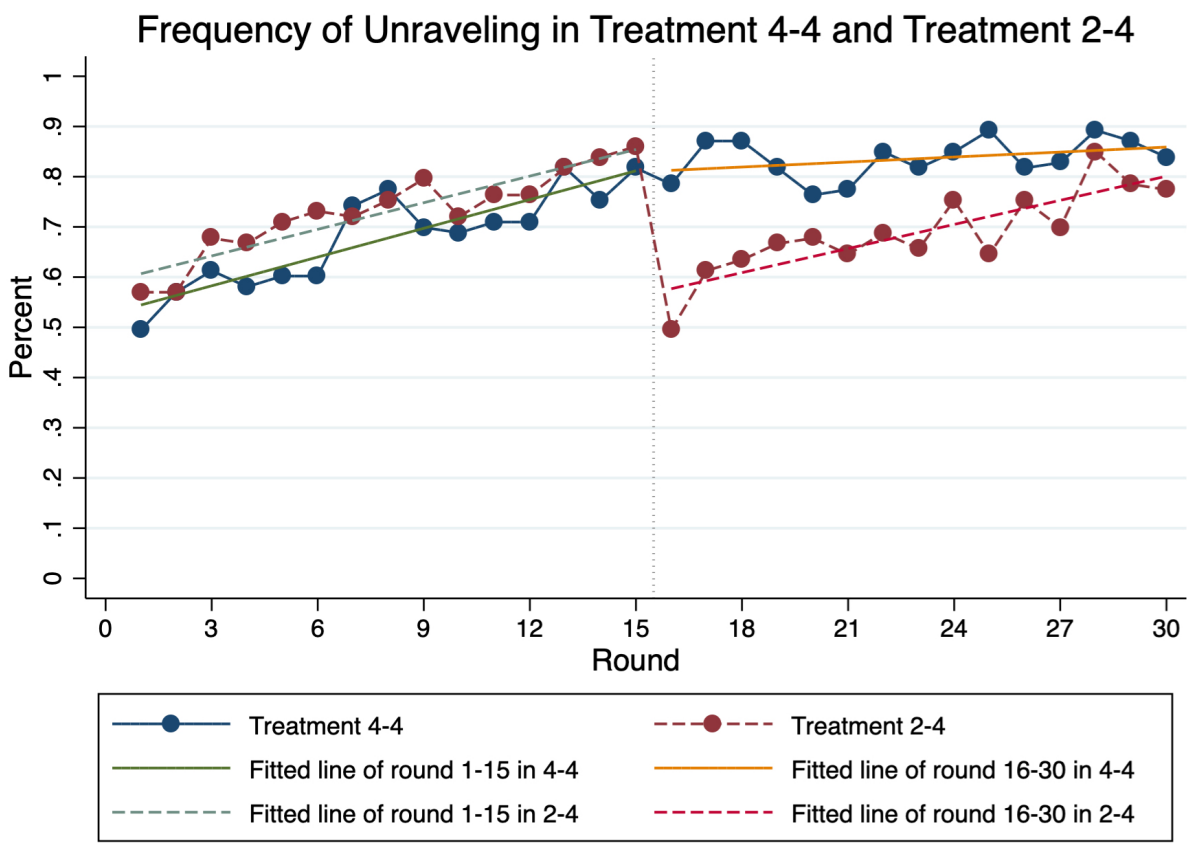

the role of the buyer in both treatments. This analysis uses the participant-role-game specific identification described in the previous section.

We can also observe evidence for learning when using the more conservative participantrole specific identification by presenting distributions of levels for the first and second 15 rounds separately. Figures 10 and 11 indicate the cumulative distributions of levels of reasoning for the first 15 rounds and the last 15 rounds in treatment 4-4 for the seller and the buyer, respectively. The cumulative distribution of levels in the last 15 rounds in treatment 4-4 lies above the cumulative distribution of identified levels in the first 15 rounds in treatment $4-4 .{ }^{14}$ We note an especially large jump for the last 15 rounds of treatment 4-4 for the highest relevant level of reasoning in buyers by almost 30 percentage points.

When applying a two-sample Kolmogorov-Smirnov test to the difference of the level distributions, we obtain a $p$-value of 0.252 for sellers, which is statistically insignificant. For buyers, the two-sample Kolmogorov-Smirnov test confirms that levels in the first 15 rounds of treatment 4-4 are significantly lower than in the last 15 rounds of treatment $4-4$ ( $p$-value $<0.001)$. One explanation is that it is relatively more difficult for the seller to learn in persuasion games. The seller moves first in this extensive-form game. Thus, she can only learn from past actions of buyers in past games (which is hampered by random rematching after each round) but not from the action of the buyer in the current game. Moreover, as prudent rationalizability refines strategies of buyers at odd levels and strategies of sellers at even levels, learning of higher order reasoning of sellers in some sense presupposes learning of higher order reasoning of buyers.

\footnotetext{
${ }^{14}$ Appendix G contains tables with numerical frequencies of levels.
} 
Figure 10: Sellers' Distribution of Levels

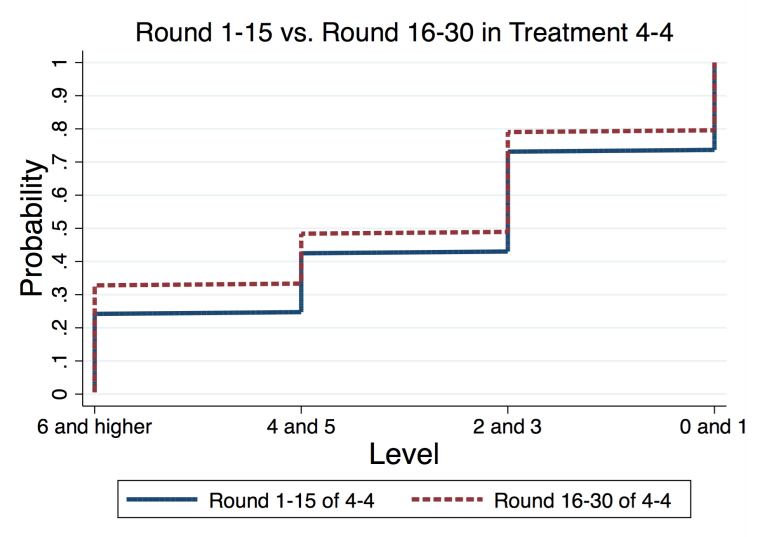

Figure 11: Buyers' Distribution of Levels

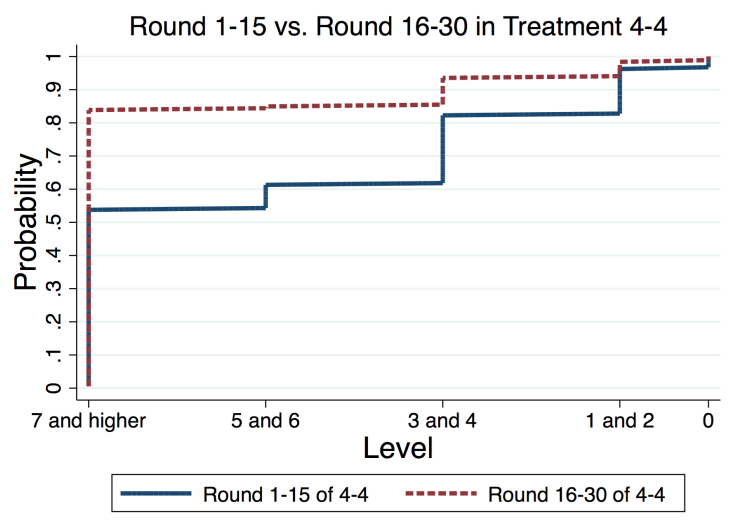

Observation 6 The cumulative distribution of levels in the last 15 rounds in treatment 4-4 first-order stochastically dominates the cumulative distribution of levels in the first 15 rounds of treatment 4-4. The difference is statistically significant only for buyers. We cannot reject Hypothesis 6 .

\subsection{Transfer of Learning}

A somewhat different picture emerges with respect to treatment 2-4. While Figure 9 still suggests that there is learning within the first and within the last 15 rounds, respectively, playing a persuasion game with two qualities in the first 15 rounds does not facilitate unraveling in the persuasion game with four qualities in the last 15 rounds. That is, learning from a simpler persuasion game is not transferred to a more complex persuasion game. In fact, the last rounds of treatment 2-4 show less unraveling than the last rounds of treatment 4-4. So playing a simple persuasion game in earlier rounds even hurts unraveling at the end. ${ }^{15}$ This is confirmed by Table 9 . The average unraveling over the last 15 rounds of treatment $2-4$ is significantly lower than average unraveling over the last 15 rounds of treatment $4-4$ ( $p$-value $<0.001)$. It is also lower than average unraveling in the first 15 rounds of treatment 2-4 although this difference is insignificant $(p$-value $=0.108)$.

Observation 7 In treatment 2-4, average unraveling is smaller in the last 15 rounds than in the first 15 rounds although the difference is not significant. Moreover, average unraveling in the last 15 rounds of treatment 2-4 is significantly smaller than average unraveling in the last 15 rounds of treatment 4-4. This is evidence for failing to transfer learning from a simpler persuasion game to a more complex persuasion game. We reject Hypothesis 7.

Figures 12 and 13 show cumulative distribution of levels for the seller and the buyer, respectively, comparing the last 15 rounds of treatment 2-4 with the last 15 rounds of treatment 4-4. In these rounds, participants play persuasion games with four quality levels in both treatments.

\footnotetext{
${ }^{15}$ We do not know what would happen though if participants were to play more than 30 rounds.
} 
Figure 12: Sellers' Distribution of Levels

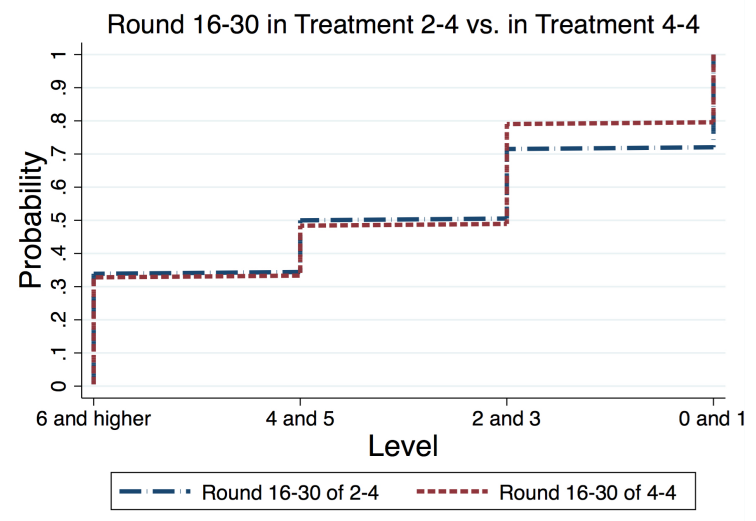

Figure 13: Buyers' Distribution of Levels

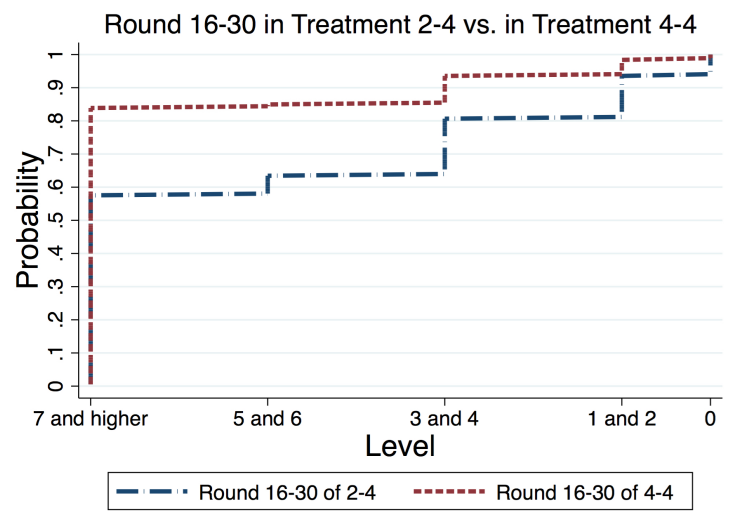

There are only minor differences for sellers as cumulative distributions of levels almost overlap from level 2 and higher. For buyers, we observe that the cumulative distribution of levels in the last 15 rounds of treatment $4-4$ first-order stochastically dominates the cumulative distribution of levels in the last 15 rounds of treatment 2-4. This is in sharp contrast to our hypothesis. Somehow, participants fail to transfer learning from the simpler to the more complex persuasion game. Buyers strongly benefit more from learning longer in the complex persuasion game than being "trained first" on a simpler persuasion game. The finding is consistent with our earlier observations on unraveling.

The visual observations are confirmed with two-sample Kolmogorov-Smirnov tests. For the sellers, the $p$-value is 0.349 . For buyers, levels in the last 15 rounds of treatment $2-4$ are significantly lower than in the last 15 rounds of treatment $4-4$ ( $p$-value $<0.001)$.

Observation 8 For sellers, the cumulative distribution of levels of reasoning in the last 15 rounds of treatment 2-4 does not first-order stochastically dominate the cumulative distribution of levels of reasoning in the last 15 rounds in treatment 4-4. Quite to the contrary, for buyers the cumulative distribution of levels of reasoning in the last 15 rounds of treatment 4-4 firstorder stochastically dominates the cumulative distribution of levels of reasoning in the last 15 rounds in treatment 2-4. We reject Hypothesis 8.

\subsection{Cognitive Ability and Reasoning}

In our sample, the average test score for the Raven's Progressive Matrices test is 16.83 out of 30. Figure 14 presents the distribution of Raven's test scores of all 372 participants. ${ }^{16}$ In the histogram, there is a large increase between scores 11 and 12. There are 45 participants whose test scores are less than or equal to 11 points comparing to 25 participants who score 12 points only. The reason of this sudden increase in frequency is not clear. One explanation might be that those participants who score only a few points tend to click through most of the test questions. Fortunately, we also record for each participant the answering time that remains

\footnotetext{
${ }^{16}$ The smoothest curve is the normal density. The less smooth curve is the kernel density.
} 
after answering each question. The more time left means that the participant used less time to answer that question. The average time left is 136.11 seconds out of 930 seconds total time. The correlation between the test core and answering time left is -0.60 implying participants with higher test score tend to spend more time on answering questions.

Figure 14: Distribution of Raven's Progressive Matrices Test Scores

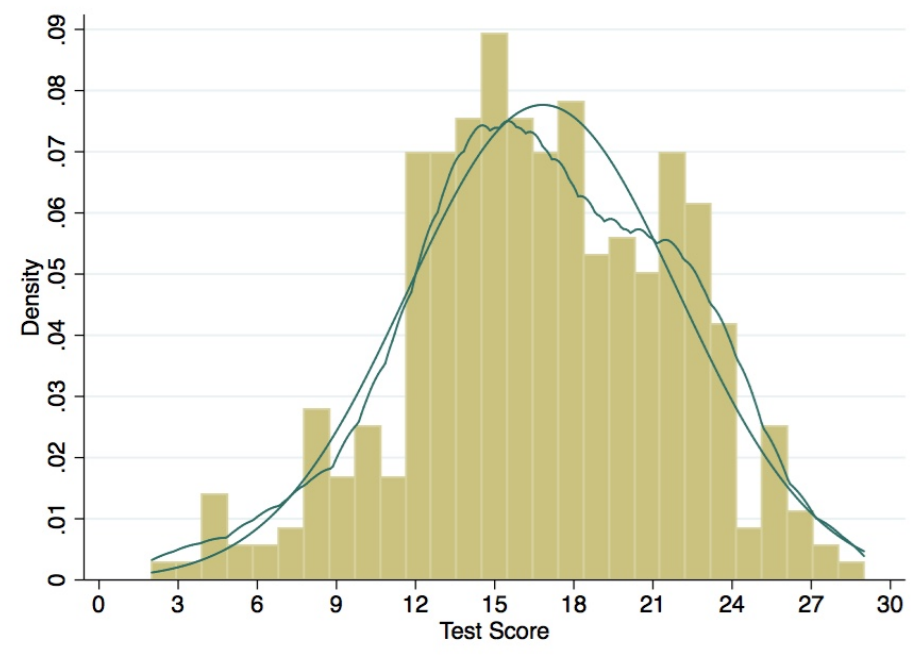

For those 45 participants with scores less than or equal to 11 points, the average time spend on each question is 11.84 seconds, and for the 7 participants who score 4 points or less, the average time is only 3 seconds. Since participants are not paid for answering the test questions, they may lack motivation to carefully complete the test. There are some participants who choose to click through most of the test questions. We do not interpret test scores as a measure of "maximal cognitive ability". Rather it might be a measure of cognitive ability as displayed at this level of (i.e., the absence of) incentives. Some participants may have higher costs of engaging their cognitive abilities than others which may contribute to their decision to complete the test with more or less care (which again resonates with recent approaches to endogeneize the cost of reasoning in level- $k$ models; see Alaoui and Penta, 2017).

In order to analyze the relationship between measured cognitive ability and reasoning, we use OLS and ordered-probit regressions of participants' levels of reasoning on Raven score, a dummy for treatment 2-4, a dummy for rounds 16-30, an interaction term of former two, a dummy indicating whether the participant self-reported experiencing difficulties in playing the game (from the questionnaire) as well as demographics. We separate the analysis by sellers and buyers because prudent rationalizability refines strategies of buyers at odd levels and strategies of sellers at even levels of reasoning.

Since the levels are discrete and OLS regressions presuppose continuous random variables, we present in Table 10 also the ordered-probit regressions in columns marked with "O-P". Columns 1 to 3 pertain to sellers; columns 4 to 6 to buyers.

The first two columns in Table 10 just differ by the including of GPA. The reason is that GPA might also reflect some degree of cognitive ability and thus be collinear with Raven test 
Table 10: Regression Results of Level of Reasoning

\begin{tabular}{|c|c|c|c|c|c|c|}
\hline & \multicolumn{6}{|c|}{ Level of Reasoning } \\
\hline & & Seller & & & Buyer & \\
\hline & OLS & OLS & $\mathrm{O}-\mathrm{P}$ & OLS & OLS & $\mathrm{O}-\mathrm{P}$ \\
\hline \multirow[t]{2}{*}{ Raven Test } & $\overline{0.0593^{* * *}}$ & $\overline{00.0584^{* * *}}$ & $\overline{00.0359^{* * *}}$ & $\overline{0.0437^{* * *}}$ & $\overline{0.0404^{* * *}}$ & $\overline{00.0343^{* * *}}$ \\
\hline & $(0.0123)$ & $(0.0123)$ & $(0.0074)$ & $(0.0113)$ & $(0.0112)$ & $(0.0088)$ \\
\hline \multirow[t]{2}{*}{ Treatment 2-4 } & $-2.5488^{* * *}$ & $-2.5342^{* * *}$ & $-1.1594^{* * *}$ & $-3.1871^{* * *}$ & $-3.1567^{* * *}$ & $-1.4797^{* * *}$ \\
\hline & $(0.0971)$ & $(0.0964)$ & $(0.0645)$ & $(0.1161)$ & $(0.1130)$ & $(0.1052)$ \\
\hline \multirow[t]{2}{*}{ Round 16-30 } & $0.2205^{*}$ & $0.2403^{* *}$ & $0.1463^{* *}$ & $0.6487^{* * *}$ & $0.6543^{* * *}$ & $0.6503^{* * *}$ \\
\hline & $(0.1206)$ & $(0.1181)$ & $(0.0730)$ & $(0.0898)$ & $(0.0909)$ & $(0.0857)$ \\
\hline \multirow{2}{*}{$\begin{array}{l}\text { Treatment } 2-4 \times \\
\text { Round16-30 }\end{array}$} & $2.5612^{* * *}$ & $2.5362^{* * *}$ & $1.1576^{* * *}$ & $2.7385^{* * *}$ & $2.7360^{* * *}$ & $1.0037^{* * *}$ \\
\hline & $(0.1531)$ & $(0.1524)$ & $(0.0977)$ & $(0.1521)$ & $(0.1539)$ & $(0.1501)$ \\
\hline \multirow[t]{2}{*}{ Time on Raven } & $0.0003^{* *}$ & $0.0003^{* *}$ & $0.0002^{* *}$ & 0.0001 & 0.0001 & 0.0001 \\
\hline & $(0.0001)$ & $(0.0001)$ & $(0.0001)$ & $(0.0001)$ & $(0.0001)$ & $(0.0001)$ \\
\hline \multirow[t]{2}{*}{ GPA } & & 0.1487 & 0.0984 & & $0.2057^{* *}$ & $0.1736^{* *}$ \\
\hline & & $(0.0967)$ & $(0.0605)$ & & $(0.0914)$ & $(0.0713)$ \\
\hline \multirow[t]{2}{*}{ Female } & $-0.2256^{* *}$ & $-0.2302^{* *}$ & $-0.1438^{* *}$ & -0.1135 & $-0.1409^{*}$ & $-0.1123^{*}$ \\
\hline & $(0.0993)$ & $(0.0989)$ & $(0.0586)$ & $(0.0844)$ & $(0.0824)$ & $(0.0679)$ \\
\hline \multirow[t]{2}{*}{ Difficult to Play } & $-0.9711^{* * *}$ & $-0.9426^{* * *}$ & $-0.5346^{* * *}$ & $-0.9864^{* * *}$ & $-0.9583^{* * *}$ & $0.6300^{* * *}$ \\
\hline & $(0.1433)$ & $(0.1465)$ & $(0.0874)$ & $(0.2074)$ & $(0.2058)$ & $(0.1222)$ \\
\hline Other Demographics & Yes & Yes & Yes & Yes & Yes & Yes \\
\hline \multirow{2}{*}{$\begin{array}{l}\text { Number of Obs. } \\
\text { (Pseudo) } R^{2}\end{array}$} & 5538 & 5464 & 5464 & 5501 & 5431 & 5431 \\
\hline & 0.3184 & 0.3194 & 0.1208 & 0.4851 & 0.4856 & 0.2244 \\
\hline
\end{tabular}

Note: Robust standard errors are clustered at the group level. Significance levels: *10\%, **5\%, and ***1\%.

scores. Also the fourth and fifth columns just differ in GPA. It turns out though that the inclusion of GPA has no affect. The correlation coefficient between Raven Test and GPA is 0.1347, which we consider as weak.

The coefficient estimate of Raven's test score is statistically significant at $1 \%$ in all regressions for both the seller and the buyer. Yet, the effect size seems to be small. In the OLS regressions for the seller and the buyer, 1 point increase in the Raven's test score is associated with just 0.0584 and 0.0404 increase in level of reasoning for the seller and the buyer, respectively. The coefficient estimate for the time spent on completing the Raven test is very small in value. It is statistically significant at $5 \%$ in the estimations for sellers and insignificant for buyers. ${ }^{17}$

Table 11 presents the marginal effects of the Raven test scores on levels of reasoning at the mean of all covariates for ordered probit. Each column represents the change in probability for

\footnotetext{
${ }^{17}$ The regression results can be viewed as a robustness check of our earlier findings. The estimates for controls in our OLS regressions confirm our findings reported earlier. The coefficient estimate of the dummy for rounds 16-30 is smaller for sellers than for buyers. This corresponds to our earlier finding that the difference in the cumulative distribution of levels between the first 15 rounds and the last 15 rounds in treatment $4-4$ is insignificant. In order to compare the difference in the cumulative distributions of levels between the last 15 rounds of treatment 2-4 and the last 15 rounds of treatment 4-4, we apply a $t$-test on the significance of the sum of coefficient estimates of the dummy for treatment 2-4 and the interaction term "treatment 2-4×Round 16-30" with the null hypothesis that $-2.5342+2.5362$ does not differ significantly from 0 . The $t$-test yields a $p$-value of 0.989 failing to reject the null hypothesis. This corresponds to our earlier finding that for sellers the cumulative distribution of levels in the last 15 rounds in treatment 2-4 is not significantly different from the last 15 rounds in treatment 4-4. For the buyer side, such a $t$-test yields a $p$-value of 0.007 suggesting that for buyers the cumulative distribution of levels in the last 15 rounds in treatment 2-4 is significantly different from the last 15 rounds in treatment 4-4.
} 
Table 11: Average Marginal Effects of Raven Test

\begin{tabular}{cc|cc}
\hline \multicolumn{2}{c|}{ Seller } & \multicolumn{2}{c}{ Buyer } \\
Level & $\mathrm{dy} / \mathrm{dx}$ & Level & $\mathrm{dy} / \mathrm{dx}$ \\
\hline \hline 1 & $-0.0066^{* * *}$ & 0 & $-0.0007^{* * *}$ \\
& $(0.0015)$ & & $(0.0003)$ \\
3 & $-0.0077^{* * *}$ & 2 & $-0.0021^{* * *}$ \\
& $(0.0017)$ & & $(0.0006)$ \\
5 & -0.0001 & 4 & $-0.0094^{* * *}$ \\
& $(0.0001)$ & & $(0.0025)$ \\
6 and higher & $0.0141^{* * *}$ & 6 & $-0.0006^{* * *}$ \\
& $(0.0029)$ & & $(0.0002)$ \\
& & 7 and higher & $0.0129^{* * *}$ \\
& & & $(0.0033)$ \\
\hline
\end{tabular}

Robust standard errors clustered at group level.

Significance levels: $* 10 \%, * * 5 \%$, and $* * * 1 \%$.

each level if there is 1 point increase in the Raven's test score. At the average, participants with 1 point higher in the Raven test score, are 1.41 and 1.29 percentage points more likely to be on the highest level of reasoning for the seller and the buyer respectively. ${ }^{18}$ We think that the effects are small, which may be due to measurement error associated with the unincentivized Raven test.

Observation 9 Levels of reasoning are significantly positively correlated with scores on Raven's progressive matrices test. Although we cannot reject Hypothesis 9, we believe that the effect is small.

\section{Discussion of Related Literature}

Jin, Luca, and Martin (2019a) run experiments on a persuasion game with both a sender and receiver. The sender knows privately which of the five states occurred. The receiver just knows that states are distributed uniformly. Incentives are such that the receiver likes to match the state and the sender prefers larger actions by the receiver. In contrast to our experiment, the decision of the seller is binary: She can choose to either report the true state or not report at all. This somewhat limits by the design levels of reasoning that could be revealed by different messages. The game is played repeatedly for 45 rounds with random rematching and role changes between rounds. There are treatments that vary in the amount of feedback that buyers receive between rounds. Reporting of the state is $95 \%$ if nature chooses states 4 or 5 , above $80 \%$ when nature chooses state 3 , and $43 \%$ when nature chooses state 2 . (Pure sequential equilibrium allows for silence when nature chooses state 1.) Thus, equilibrium predicts a substantial fraction if not most of the seller's behavior. The authors state that instances in which sellers do not report even though they should according to theoretical predictions are due to insufficient skepticism on part of the receivers about non-disclosure. Our approach suggests a more nuanced interpretation: Skepticism alone is not sufficient for unraveling since otherwise any first-level

\footnotetext{
${ }^{18}$ Note that the sum of marginal effects must be zero. If there is 1 point increase in the Raven test score at the mean, the distribution shifts to the right slightly. It shifts mass out of the lower levels.
} 
prudent rationalizable strategy would imply unraveling already. Higher-order reasoning alone is also not sufficient for unraveling. This follows from applying extensive-form rationalizability à la Pearce (1984) and Battigalli (1997) to the game. Similar to prudent rationalizability, extensiveform rationalizability involves higher-order beliefs but different from prudent rationalizability it entails no skepticism. It does not yield unraveling outcomes. We conclude that unraveling requires both, higher-order reasoning with at least some small amount of skepticism. Jin, Luca, and Martin (2019a) also observe that feedback facilitates some learning towards unraveling. This corresponds somewhat to our observation that learning facilities unraveling.

Hagenbach and Perez-Richet (2018) investigate experimental results with verifiable information in a number of sender-receiver games that vary by the alignment of incentives between the sender and receiver. This is somewhat different from our experiment in that we focus on the standard case in which the seller's equilibrium payoff is increasing in quality level and receivers like to match the quality. Hagenbach and Perez-Richet (2018) study how actions of players change when facing incentives of different complexity. In contrast, we slightly vary the complexity of the sender's action space but keep the incentive structure fixed, which allows us to get a glimpse of the participants' strategic sophistication. In their last version of the paper, Hagenbach and Perez-Richet (2018) also introduce an iterative solution concept, namely, iterated elimination of obviously undominated strategies. They observe that this solution concept organizes their data well. As Hagenbach and Perez-Richet (2018) observe, our more standard disclosure game does not belong to the class of disclosure games studied in their paper. So our results are complementary.

In a very recent experiment, Deversi, Ispano, and Schwardmann (2019) study the effect of precise versus vague disclosure rules. Essentially they compare two treatments: One treatment is more or less a standard persuasion game in which the sender can send any message as long as it is verifiable information. The other treatment requires as in Jin, Luca, and Martin (2019a) the sender to disclose precisely her private information when she decides to disclose any information. Clearly, the theoretical prediction in both versions of the persuasion game is unraveling. They show that imposing precise disclosure leads to less but more precise disclosure that is especially helpful to naïve receivers.

Perhaps the first experiment on disclosure of verifiable information is Forsythe, Isaac, and Palfrey (1989). They test a series of experimental first-price auctions where sellers have common value information about the good and decide whether to reveal this information to bidders (who in one treatment have a private value component as well) before the auction. There are several Bayes-Nash equilibria among which the skeptical sequential equilibrium induces unraveling. They report that participants converge to this equilibrium over a number of repetitions.

King and Wallin (1991) study disclosure of verifiable information by sellers in double auction markets. They observe convergence to full disclosure except in markets where buyers did not know the sellers' options do disclose information or where a large number of disclosure strategies were available. Our work suggests that such failure of convergence to full disclosure may be due to lack of reasoning when buyers do not know the sellers' disclosure options or levels of reasoning insufficient for obtaining full disclosure when faced with many disclosure options.

In a recent "companion" experiment, we study experimentally the effect of unawareness in persuasion games ( $\mathrm{Li}$ and Schipper, 2018). Unawareness refers to the lack of conception 
rather than the lack of information. This is relevant in persuasion because the buyer can infer something from the silence of the seller only if he is aware of what the seller may possibly know. Prudent rationalizability predicts unraveling in persuasion games with full awareness but failure of unraveling in persuasion games with unawareness (Heifetz, Meier, and Schipper, 2019a; see also Schipper and Woo, 2019, for an application to electoral campaigning). We test this prediction experimentally and find some support for it.

In a slightly different disclosure game with mandatory disclosure, Jin, Luca, and Martin (2019b) study whether participants in an experiment would choose complex disclosures over more simple ones. While in their game the sender must disclose, she has the discretion with regard to the level of complexity of the disclosure message. They find that senders frequently use complex disclosures to their profit despite the fact that receivers' stated beliefs reveal that they understand the senders' motivation for obfuscation. Yet, receivers seem to be overconfident with regard of their ability to correctly read complex reports.

Although it is not a persuasion game, Benndorf, Kübler, and Normann (2015) test the voluntary revelation of private verifiable information in a labor-market experiment. Workers can costly reveal their productivity or not (i.e., binary action). Participants take only the role of workers (i.e., sellers). If they reveal, then they earn their productivity minus revelation cost. Otherwise, they earn average productivity of workers who do not reveal. There is no unraveling in the sense of persuasion. Employers do not explicitly deduce information from non-revelation. Essentially it is a complete information game in which one action of the players can be interpreted as disclosing verifiable information. The authors find that participants reveal less frequent than predicted in Nash equilibrium, especially with respect to low-productivity workers. Using the strategy method but the same game, Benndorf, Kübler, and Normann (2017) report a level- $k$ analysis. They find 30 percent naive players and 25 percent play consistent with level 4 or higher with the most frequently observed levels being 2 and 3 .

Our work is related to the literature on level- $k$ reasoning. Starting with Nagel (1995) and Stahl and Wilson (1995), many experimental studies have been devoted to infer levels of strategic reasoning from behavior in experimental games using the level- $k$ model or cognitive hierarchies (see Crawford, Costa-Gomes, and Iriberri, 2013, for a survey). Level- $k$ reasoning models differ from iterated admissibility or prudent rationalizability used in our paper. Under prudent rationalizability, any strategy is zero-level rationalizable. At level-1, a strategy is prudent rationalizable if there exists a full support belief over opponents strategies with which this strategy is optimal. A strategy is level-2 prudent rationalizable if there exists a full support belief over opponents' level-1 prudent rationalizable strategies with which this strategy is optimal etc. For level- $k$ reasoning, the level-0 strategy is fixed to some more or less ad hoc strategy. A level-1 player best responds to this level-0 strategy. A level-2 player best responds to the level-1 players (or some mixture of level-0 and level-1 players in the case of the cognitive hierarchy model by Camerer, Ho, and Chong, 2004) etc.

The identification of levels of reasoning in level- $k$ reasoning depends heavily on first-level beliefs or equivalently the behavior assumed for "level-0" types. Assumptions on first-level beliefs seem to have been largely motivated by modeling either maximal unpredictable behavior (e.g. Wilson and Stahl, 1995) or "non-strategic" behavior (for a stark example, see Arad and Rubinstein, 2012). In the case of our persuasion game, it is not clear to us what would be the "most natural" assumption. If we subscribe to the paradigm that first-level beliefs/level-0 types 
should model "non-strategic" behavior, then naive truthful disclosure on part of sellers seems to be the "most natural" assumption but also the most uninteresting one because it implies unraveling at level 1 (i.e., level-0 for sellers and level-1 for buyers). This is true more generally for level- $k$ reasoning. Any normal-form rationalizable outcome can be made consistent with level-1 reasoning by simply assuming that level-0 chooses strategies yielding the rationalizable outcome (see Schipper and Zhou, 2019, for a rigorous argument). This emphasizes the usefulness of interesting extra-theoretical assumptions on level-0 behavior for identifying levels of reasoning.

The question about the most natural and useful assumption about level-0 behavior is not the only impediment to applying level- $k$ reasoning to persuasion games. Although level- $k$ thinking has been previously applied to extensive-form games (e.g, Crawford, 2003, Ho and Su, 2013), it is a normal-form solution concept. In contrast, persuasion games are extensive-form games. In extensive-form games, it is natural to allow players to update their beliefs about opponents' levels conditional on information sets reached in the game. Such updates are not considered in level- $k$ reasoning. More recently, Schipper and Zhou (2019) extend the definition of level$k$ reasoning to allow for extensive-form reasoning in extensive-form games and show various properties of this solution concept. This extensive-form version of level- $k$ reasoning is still too weak in persuasion games because unraveling requires some form of skeptical beliefs on part of the buyers about the true quality. This skepticism we have "for free" with cautious (i.e., full-support) beliefs featured in prudent rationalizability. ${ }^{19}$

Typically experimental studies on level- $k$ thinking fit empirical distributions of level- $k$ types to behavior. While it has mostly been applied to simultaneous move games (e.g., Nagel 1995, Stahl and Wilson 1995, Costa-Gomes, Crawford, and Broseta 2001, Camerer, Ho, and Chong 2004, Costa-Gomes and Crawford 2006, Arad and Rubinstein 2012), Crawford (2003) applied it to sender-receiver games (without verifiable information) and explained over-communication and systematic deception. Ho and $\mathrm{Su}$ (2013) apply level- $k$ to a dynamic game where players choose rules based on their best guesses of others' rules and use historical plays to improve their guesses. A rough conclusion from the literature is that the majority of players are best fitted to level-1 and level-2 reasoning and there is rarely evidence for level-4 reasoning or higher in experimental data. See Crawford, Costa-Gomes, and Iriberri (2013) for a comprehensive survey on applications of level- $k$ model.

Brandenburger, Danieli, and Friedenberg (2019) study the problem of identifying levels of reasoning about rationality in extensive-form games. They characterize the class of extensiveform games in which they can identify $k$-level strong belief in rationality. In contrast to the level- $k$ literature, they are interested in avoiding ad-hoc assumptions on first-levels beliefs (or "level-0" behavior). And in contrast to our approach, they also avoid implicit assumptions on the "richness" of type spaces and instead allow for any type space. This is very appropriate for experiments as experimenters never know with which initial beliefs participants arrive for the experiment. Unfortunately, their approach does not apply to our setting. First, the persuasion game we study does not satisfy their assumptions. In particular, it is not satisfy their genericity assumption. Thus, their identification result does not apply to our games. Second, the solution concept they consider is too weak in persuasion games. They consider extensive-form rationalizability, or more generally extensive-form best response sets (Battigalli and Friedenberg,

\footnotetext{
${ }^{19}$ If prudent rationalizability is replaced with the closest analogue to it that does not feature caution, which is extensive-form rationalizability (Pearce, 1984, Battigalli, 1997), then unraveling does not occur.
} 
2012) while we consider iterated admissibility/prudent rationalizability. For unraveling, the buyer must have skeptical beliefs about the quality and put some weight on the worst quality consistent with the message. Full-support beliefs featured in prudent rationalizability imply such skepticism automatically. But with extensive-form rationalizability and extensive-form best response sets, the buyer may not put any weight on the worst quality. Consequently, it is possible that the seller discloses also qualities worse than her actual one believing that the buyer believes in the best quality that is disclosed.

There is also a small but growing literature that applies cognitive tests to analyze the relationship between cognitive ability and strategic behavior (e.g., Burnham et al. 2009, Oechssler et al. 2009, and Brañas-Garza et al. 2012, Benito-Ostolaza et al. 2016). To measure each subject's cognitive abilities, we use Raven's standard progressive matrices test (Raven et al. 2000). It is a test of cognitive abilities that can be easily administered. It is nonverbal, requires no reading, writing, or mathematical training, thus making it feasible to use in a wide variety of contexts, and the results are comparable between participants of different backgrounds. In economics, Raven test scores have been found to correlate positively with fewer Bayesian updating errors (Charness et al., 2011) and with more accurate beliefs (Burks et al., 2009). In a recent study, Gill and Prowse (2016) apply a level- $k$ framework to analyze individual's experimental behavior in a $p$-beauty contest game. They find a positive relationship between cognitive abilities and participants' level of reasoning. Bhatt and Camerer (2005) and Coricelli and Nagel (2009) use fMRI to make inferences about cognition and level- $k$ reasoning.

\section{A Sequential Equilibrium}

Milgrom and Roberts (1986) observe that there is a sequential equilibrium in the persuasion game such that the buyer believes at information set $Q^{\prime} \in 2^{Q} \backslash\{\emptyset\}$ that the quality selected by nature is $\min Q^{\prime}$. In this sense, the buyer adopts a skeptical posture in this equilibrium. Subsequently his equilibrium action at information set $Q^{\prime}$ is $x^{*}=\min Q^{\prime}$. The seller's equilibrium strategies are such that if the quality level selected by nature is $q$ then $\sigma_{s}(q)$ with $q=\min \sigma_{s}(q)$. Thus, sequential equilibrium predicts unraveling but it does not allow us to infer information about levels of reasoning.

Proposition 2 Any pure sequential equilibrium of the persuasion game with two or four qualities must be consistent with the actions described in Tables 12 and 13, respectively. Consequently, any pure sequential equilibrium is unraveling.

The proof of the proposition is a corollary of Milgrom and Roberts (1986, Proposition 2) upon noticing that both the persuasion game with two and the game with four quality levels satisfy the conditions of their proposition. A minor difference is that we restrict quantities to a finite set while Milgrom and Roberts (1986) allow quantities to be any nonnegative number. This difference is immaterial to pure equilibrium in our game considered here.

The last columns of Tables 12 and 13 show the actions of the buyer. I.e., for any the seller's action indicated in the preceding columns, the equilibrium action of the buyer is written in the last column in the row. 

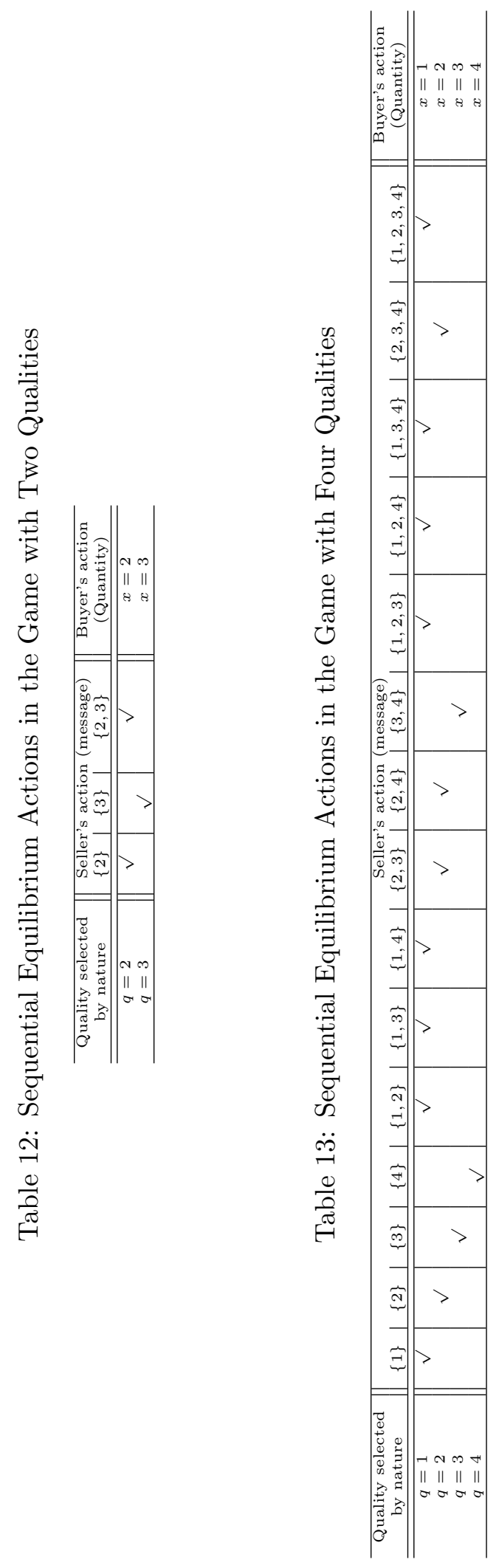
Figure 15: Frequencies of Sequential Equilibrium and Outcomes Consistent with $k$-Level Prudent Rationalizability by Treatment
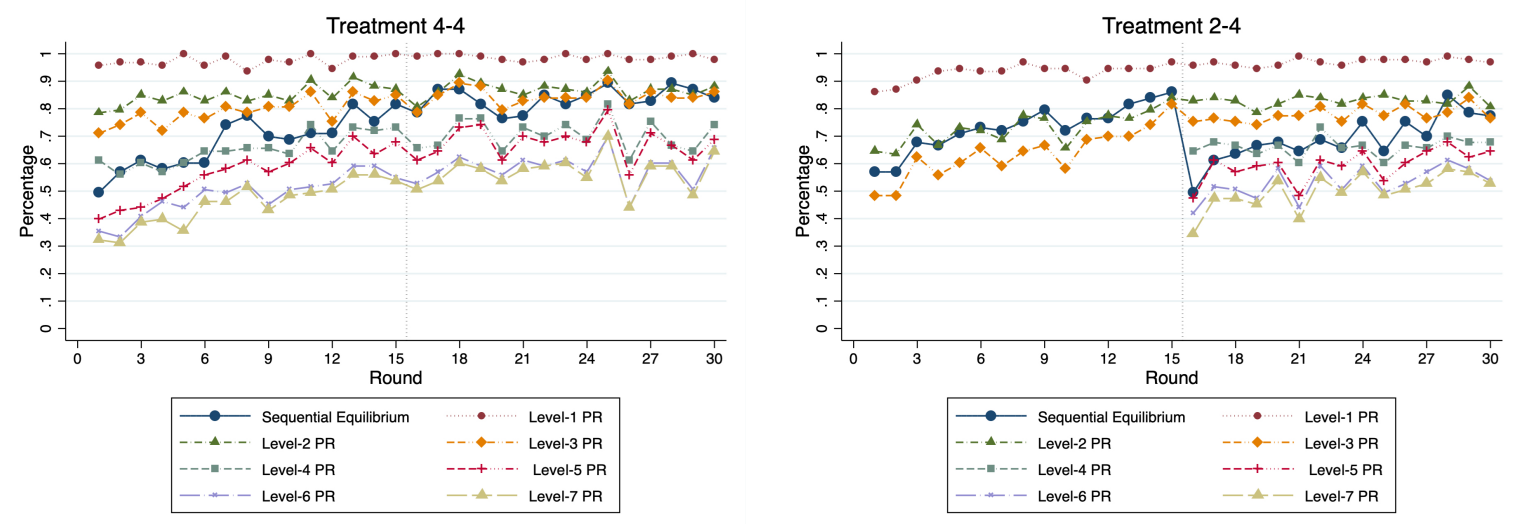

Note that prudent rationalizability slightly refines the seller's sequential equilibrium strategies. For instance in the persuasion game with four qualities, when the quality selected by nature is $q<4$, the message $\{q\}$ is part of a sequential equilibrium while it can not part of any prudent rationalizable strategy. It is consistent though with at most one level of reasoning in the prudent rationalizable procedure. Another difference between the solution concepts is for instance that when the quality selected by nature is $q<3$, then $\{q, y\}$ for $y>q, y \in\{2,3,4\}$, is part of a sequential equilibrium while it is not part of any prudent rationalizable strategy. It is consistent though with at most two levels of reasoning in the prudent rationalizability procedure.

When nature selects $q=1$, then sequential equilibrium allows for any message containing $q=1$. In such a case, it has no predictive power. This is in contrast to prudent rationalizability, which uniquely predicts message $\{1,2,3,4\}$ in this case. When nature selects $q=4$, then the prudent rationalizable action of the seller coincides with her sequential equilibrium action. The refinement power of prudent rationalizability stems from the prudence or caution embodied in the solution concept. For buyers, the set of pure sequential equilibrium quantities coincide with prudent rationalizable quantity choices.

Since prudent rationalizability is a refinement of pure sequential equilibrium in our games, it is not surprising that more actions are consistent with pure sequential equilibrium than with prudent rationalizability. Yet, since prudent rationalizability yields a prediction for every finite level of reasoning, we can compare sequential equilibrium to $k$-level prudent rationalizability for all relevant levels. Figure 15 (left figure) shows the frequencies of sequential equilibrium outcomes and outcomes consistent with $k$-level prudent rationalizability in treatment 4-4 over all 30 rounds. We observe that initially about $50 \%$ of all outcomes are consistent with sequential equilibrium. This increases to about $80 \%$ after 15 rounds and more or less stays there for the remaining rounds. Initially, the fit of sequential equilibrium is similar to outcomes consistent with at most 4-level prudent rationalizable strategies. After about 15 rounds, the fit becomes similar to outcomes consistent with at most 3-level prudent rationalizable strategies. A similar picture emerges for treatment 2-4 (Figure 15, right figure). For the last 15 rounds, when players 
also play the persuasion game with four quality levels, the stylized facts are the same as for the first 15 rounds of treatment 4-4. Similarly, in the first 15 rounds, when players play the persuasion game with two quality levels only, about $50 \%$ of all outcomes are consistent with sequential equilibrium. This also increases to about $80 \%$ towards the end of the first 15 periods. We observe that initially sequential equilibrium is similar to outcomes consistent with two to three levels of reasoning of prudent rationalizable strategies, while it is clearly similar to 2-level prudent rationalizability towards the end of the first 15 periods. Thus, overall no matter whether players play a persuasion game with two or four quality levels, the fit of sequential equilibrium is somewhere in the middle of levels of reasoning relevant for those games, respectively. It is as if sequential equilibrium captures some intermediate "effort" of strategic reasoning in those games.

\section{B Proof of Proposition 1}

A shorter inductive proof could be stated. Yet, since we deal with at most four quality levels and we need to explicitly identify levels of reasoning in the experimental data, we find it more useful to prove the proposition by exhaustion and work out strategies level-by-level.

Level 1: For a buyer who receives message $M$, any level-1 prudent rationalizable strategy involves him choosing any quantity which is a best response to some full support belief over qualities in $M$. Any quantity choice that is not equal to some quality $m$ in $M$ is strictly dominated by some quantity choice that is a best response to some quality in $M$. In particular, if the buyer receives message $\{q\}$, then he knows that the quality is $q$ and hence he purchases $x=q$.

Any seller's strategy is level-1 prudent rationalizable because her beliefs about buyer's behavior are not yet constrained in any way (except for being full support).

Level 2: If the quality of the seller is $q$, then any message $M$ with $\{s, q, r\} \subseteq M$ for $s \leq$ $q<r$ is level-2 prudent rationalizable with the full support belief over buyer's level-1 prudent rationalizable strategies that puts sufficiently large probability on the buyer buying $r$ upon receiving message $M$ while buying $q$ upon receiving any other message consistent with $q$. (This case obviously applies only to $q<\max Q$.) Any non-singleton message $M$ with $\max M=q$ (e.g., $M=\{r, q\}$ with $r<q$ ) is not level-2 prudent rationalizable for the seller with quality $q$. To see this, note that given a full support belief over buyer's strategies, this message yields a strictly smaller expected payoff than message $\{q\}$. In particular, this means that if $q=4$, $M$ is (uniquely) level-1 prudent rationalizable for the seller if and only if $M=\{4\}$. If $q<4$, then any singleton message consistent with $q$ is not level-2 prudent rationalizable for the seller because with a full support belief over level-1 prudent rationalizable strategies of the buyer, any message $\{q, 4\}$ yields a strictly larger expected payoff.

Any level-1 prudent rationalizable strategy of the buyer is also level-2 prudent rationalizable for the buyer.

Level 3: A buyer who receives a non-singleton message $M$ with $\max M=q$ knows from the seller's level-2 prudent rationalizable strategies that the quality selected by nature is not $q$. 
Hence, any level-3 prudent rationalizable strategy of the buyer must prescribe to buy a quantity $r<q$ in this case. In particular, if $M=\{q, 4\}$, then any level-3 prudent rationalizable strategy of the buyer must prescribe to buy $q$.

Any level-2 prudent rationalizable strategy of the seller is also level-3 prudent rationalizable.

Level 4: For a seller with quality $q=3$, the only level-4 prudent rationalizable strategy is to send message $\{3,4\}$. Any other message consistent with $q=3$ and level-2 prudent rationalizable strategies of the seller yields a strictly lower expected payoff because there is strict positive probability that the buyer buys a quantity strictly less than 3 .

For a seller with quality $q<3$, any strategy prescribing a non-singleton message $M$ with $\max (M \backslash \max M)=q$ (i.e., the second highest quality being $q$ ) is not level-4 prudent rationalizable. To see this, note that message $\{q, \ldots, 4\}$ yields a strictly higher expected payoff than any such $M$ for any full support belief over buyer's level-3 prudent rationalizable strategies. It implies that for $q<3$, any strategy with $M(q)=\{q, y\}$ with $y>q$ is not prudent rationalizable at level 4 . This is because the buyer knows with such a message that the quality is not $y$ and hence it must be $q$. The seller can improve the expected payoff by instead selecting a message $M(q)$ with $|M(q)| \geq 3$. Any other level-3 prudent rationalizable strategy of the seller is also level-4 prudent rationalizable by the same arguments as in the characterization of level- 2 prudent rationalizable strategies.

Any level-3 prudent rationalizable strategy of the buyer is also level-4 prudent rationalizable.

Level 5: A buyer who receives a message $M$ with $|M| \leq 3$ knows from the seller's level-4 prudent rationalizable strategies that $q=\min M$. To see this note that if $q=4$ then any level-4 prudent rationalizable strategy prescribes message $\{4\}$, if $q=3$ then it prescribes message $\{3,4\}$, and if $q=2$, then any level- 4 prudent rationalizable strategy prescribes $\{2,3,4\}$, or $\{1,2,3,4\}$. When $q=1$, then any level-4 prudent rationalizable strategy prescribes $\{1,2,3\}$, $\{1,2,4\},\{1,3,4\}$, or $\{1,2,3,4\}$. For any other message, any purchase consistent with level-4 prudent rationalizable strategies of the buyer is also consistent with level-5 prudent rationalizable strategies of the buyer.

Any 4-level prudent rationalizable strategy of the seller is also level-5 prudent rationalizable.

Level 6: For a seller with quality $q=2$, any level-6 prudent rationalizable strategy must prescribe to send a non-singleton message $M$ with $\min M=2$. To see this, note that the only level-4 prudent rationalizable strategy with $\min M=1$ of a seller with $q=2$ is $\{1,2,3,4\}$. Given a full support belief over buyer's level-5 prudent rationalizable strategies, any other level-4 prudent rationalizable strategy of the seller yields a higher expected payoff. For a seller with quality $q=1$, any level-6 prudent rationalizable strategy must prescribe to send message $\{1,2,3,4\}$ because if the seller sends any other message $M$, then $|M| \leq 3$ and thus the buyer knows that the quality selected by nature is 1 .

Any level-5 prudent rationalizable strategies of the buyer are also level-6 prudent rationalizable.

Level 7: The buyer, upon receiving message $\{1,2,3,4\}$ knows from level- 6 prudent rationalizable strategies of the seller that $q=1$. In fact, for any message $M$ with $1 \in M$, the buyer now 
knows that $q=1$. Consequently any level-7 prudent rationalizable strategy of the buyer must prescribe to buy one unit upon receiving a message $M$ with $1 \in M$.

Any level-6 prudent rationalizable strategies of the seller are also level-7 prudent rationalizable.

No further refinements of strategies occur at higher levels of the prudent rationalizability procedure. 


\section{Experimental Instructions}

\section{C.1 Instructions for Treatment 4-4}

\section{Instructions for the Market Game}

Welcome to the experiment!

Please now turn off your cell phones and any other electronic devices. These must remain turned off for the duration of the experiment. The amount of money you will earn in this experiment will depend on your choices. Thus, it is in your best interest to follow these instructions carefully. You will be paid in cash at the end of the experiment. During the experiment, we ask that you please do not talk to each other. If you have any question, please raise your hand and an experimenter will assist you.

The experiment is made up of 3 phases. The first phase consists of a repeated market game. In the second phase you will complete a simple test. The third phase consists of a questionnaire.

\section{Phase 1}

The market game in the first phase is repeated for 30 rounds. In each round you will be randomly selected as a seller or buyer and then paired up with another participant in the other role. Your role assignment is shown to you on the computer screen through each round. The market works as follows:

Each market consists of one seller and one buyer. The seller can sell an imaginary object with a fixed price of $\$ 4$ to the buyer. The object's quality may differ. The quality is randomly chosen from 1, 2, 3, and 4 with equal probability by the computer. 4 represents the highest quality while 1 is the lowest quality. At the beginning of each round, the seller is notified of the object's quality $(q)$, which is displayed on the computer screen. The seller is able to supply as many objects of that quality as demanded by the buyer. The buyer does not know the object's quality unless the seller chooses to provide some information about the quality to the buyer. The seller can communicate through the computer any set of qualities to the buyer provided that the true quality is contained in this set. For instance, if the true quality of the object is 2 , then the seller can send the buyer one of the 8 messages from the right-hand side column of the following table. The associated messages you will see on the computer screen are displayed in the column on the left. Out of 4 numbers, the shaded number(s) is(are) contained in the message. So if the true quality is 2 , any possible message sent by the seller must include the true quality 2 . 


\begin{tabular}{|c|c|c|c|c|}
\hline 1 & 2 & 3 & 4 & "The quality is either $1,2,3$, or $4 . "$ \\
\hline 1 & 2 & 3 & 4 & "The quality is either 1,2 , or $3 . "$ \\
\hline 1 & 2 & 3 & 4 & "The quality is either 1,2 , or $4 . "$ \\
\hline 1 & 2 & 3 & 4 & "The quality is either 2,3 , or $4 . "$ \\
\hline 1 & 2 & 3 & 4 & "The quality is either 1 or $2 . "$ \\
\hline 1 & 2 & 3 & 4 & "The quality is either 2 or $3 . "$ \\
\hline 1 & 2 & 3 & 4 & "The quality is either 2 or $4 . "$ \\
\hline 1 & 2 & 3 & 4 & "The quality is $2 . "$ \\
\hline
\end{tabular}

After receiving the information, the buyer selects the quantity of the good $(x)$ to purchase. The quantity to purchase is restricted to $1,2,3$, and 4 in the experiment. So only one of these 4 integers is acceptable as the buyer's purchasing quantity.

The seller's payoff in each round is the price of the object (\$4) multiplied by the number of units $(x)$ sold to the buyer:

$$
4 \cdot x
$$

The buyer's payoff in each round is determined by both the quantity purchased $(x)$ and the true quality $(q)$ of the object:

$$
12-6 \cdot \sqrt{|x-q|}+6 \cdot x^{\frac{2}{3}} \cdot q^{\frac{1}{3}}-4 \cdot x .
$$

The key to interpret the buyer's payoff function is that for each quality $q$ the buyer's payoff is maximized when the units purchased is equal to the true quality, that is $x=q$. We realize that this formula may look complicated. You may want to look at the following payoff table instead. The entries in the table show your rounded payoff for each true quality level (in columns) and units purchased (in rows). For instance, if the true quality is $q=4$ and you purchase 2 units (that is, $x=2$ ), then your payoff in this round is approximately $\$ 11$.

\begin{tabular}{c|c|c|c|c|c|}
\multicolumn{1}{c|}{} & \multicolumn{5}{c}{ Quality } \\
\cline { 3 - 7 } \multicolumn{1}{c|}{} & $q=1$ & $q=2$ & $q=3$ & $q=4$ \\
\cline { 2 - 6 } Units & $x=1$ & 14 & 10 & 8 & 7 \\
\cline { 2 - 6 } Purchased & $x=2$ & 8 & 16 & 12 & 11 \\
\cline { 2 - 6 } & $x=3$ & 4 & 10 & 18 & 14 \\
\cline { 2 - 6 } & $x=4$ & 1 & 7 & 12 & 20 \\
\hline
\end{tabular}


After the buyer informs the seller about the quantity purchased via the computer, the computer will show the seller and the buyer the quantity purchased, the true quality and their own payoffs for the round just played. For an instance, if the true quality is 4 and the buyer chooses to purchase 3 units, then the seller's payoff is $\$ 12$ and the buyer's payoff is $\$ 14$.

The experiment proceeds to the next round after both the seller and the buyer acknowledge this information by clicking the button on the computer screen. In the next round, each participant again is randomly selected to be a buyer or a seller and randomly matched with some participant of the experiment to play the market game. The true quality of the seller in this market game is also randomly selected and may differ from the true quality of the prior round. Phase 1 ends after 30 rounds of the market game have been played.

\section{Phase 2}

Phase 2 consists of a simple test. The test is made up of 30 questions. For every question, there is a pattern with a piece missing and a number of pieces below the pattern. You have to choose which of the pieces below is the missing one to complete the pattern. For each question, one and only one of these pieces is the missing one to complete the pattern. You will score 1 point for every correct answer. After completing the test, you will be informed of your own test score. The test score will not affect your payment that you receive from the experiment.

After completing both phases 1 and 2, your cash payment will be displayed on your

computer screen. Your cash payment will be your payoff from one round randomly drawn from the 30 rounds of the market game plus a $\$ 5$ show-up fee.

\section{Phase 3}

While waiting to be called upon for payment, please complete the questionnaire that the experimenter will hand you. The questionnaire contains questions about demographics. Please carefully complete this questionnaire as this information is very important to us. After completing the questionnaire, please remain in your seat until you have been called upon for payment.

Thank you very much for your participation. 


\section{C.2 Instructions for Treatment 2-4}

\section{Instructions for the Market Game}

Welcome to the experiment!

Please now turn off your cell phones. These must remain turned off for the duration of the experiment. The amount of money you will earn in this experiment will depend on your choices. Thus, it is in your best interest to follow these instructions carefully. You will be paid in cash at the end of the experiment. During the experiment, we ask that you please do not talk to each other. If you have any question, please raise your hand and an experimenter will assist you.

The experiment is made up of 3 phases. The first phase consists of a repeated market game. In the second phase you will complete a simple test. The third phase consists of a questionnaire.

\section{Phase 1}

The market game is repeated for 30 rounds. In each round you will be randomly selected as a seller or buyer and then paired up with another participant in the other role. Your role assignment is shown to you on the computer screen as the experiment proceeds. The market works as follows:

Each market consists of one seller and one buyer. The seller can sell an imaginary object with a fixed price of $\$ 4$ to the buyer. The object's quality may differ round by round which is randomly chosen from a set of numbers. At the beginning of each round, the seller is notified of the objects's quality $(q)$, which is displayed on the computer screen. The seller is able to supply as many objects of that quality as demanded by the buyer. The buyer does not know the object's quality unless the seller chooses to provide some information about the quality to the buyer. The seller can communicate through the computer any set of qualities to the buyer provided that (s)he does not exclude the true quality.

In the first 15 rounds, the quality is randomly chosen from 2 and 3 with equal probability by the computer. 3 represents the higher quality while 2 is the lower quality. For instance, if the true quality is 2 , then the seller can send one the the following 2 messages shown in the right-hand side column of the following table to the buyer. The images in the column on the left are the associated messages displayed on the computer screen. The shaded number(s) is(are) contained in the message. So if the true quality is 2 , any 


\begin{tabular}{|l|l|l|l|}
\hline 2 & 3 & & "The quality is either 2 or $3 . "$ \\
\hline 2 & 3 & & "The quality is $2 . "$ \\
\hline
\end{tabular}

possible message sent by the seller must include the true quality 2 .

For the remaining of 15 rounds, the quality is randomly chosen from 1, 2, 3, and 4 with equal probability by the computer. 4 represents the highest quality while 1 is the lowest quality. For instance, if the true quality of the object is 2 , then the seller can send the buyer one of the 8 messages from the right-hand side column of the following table. The associated messages that you will see on the computer screen are displayed in the column on the left. Out of 4 numbers, the shaded number(s) is(are) contained in the message. So in this case, any possible message sent by the seller must include the true quality 2.

\begin{tabular}{|c|c|c|c|c|}
\hline 1 & 2 & 3 & 4 & "The quality is either $1,2,3$, or $4 . "$ \\
\hline 1 & 2 & 3 & 4 & "The quality is either 1,2 , or $3 . "$ \\
\hline 1 & 2 & 3 & 4 & "The quality is either 1,2 , or $4 . "$ \\
\hline 1 & 2 & 3 & 4 & "The quality is either 2,3 , or $4 . "$ \\
\hline 1 & 2 & 3 & 4 & "The quality is either 1 or $2 . "$ \\
\hline 1 & 2 & 3 & 4 & "The quality is either 2 or $3 . "$ \\
\hline 1 & 2 & 3 & 4 & "The quality is either 2 or $4 . "$ \\
\hline 1 & 2 & 3 & 4 & "The quality is $2 . "$ \\
\hline
\end{tabular}

After receiving the information, the buyer selects the quantity of the good $(x)$ to purchase. The quantity to purchase is restricted to $1,2,3$, and 4 and only one of these 4 integers is acceptable as the buyer's purchasing quantity.

The seller's payoff in each round is the price of the object $(\$ 4)$ multiplied by the number of units $(x)$ sold to the buyer:

$$
4 \cdot x
$$

The buyer's payoff in each round is determined by both the quantity purchased $(x)$ and the true quality $(q)$ of the object:

$$
12-6 \cdot \sqrt{|x-q|}+6 \cdot x^{\frac{2}{3}} \cdot q^{\frac{1}{3}}-4 \cdot x .
$$


Don't panic! Here is what it means: for each quality $q$ the buyer's payoff is maximized when the units purchased is equal to the true quality, that is $x=q$. Instead of looking at the formula, it would be easier to look at the following payoff table. The entries in the table show your rounded payoff for each true quality level (in columns) and units purchased (in rows). For instance, if the true quality is $q=4$ and you purchase 2 units (that is $x=2$ ), then as the buyer your payoff in this round is approximately $\$ 11$.

\begin{tabular}{|c|c|c|c|c|c|}
\hline & \multicolumn{4}{|c|}{ Quality } \\
\hline & & $q=1$ & $q=2$ & $q=3$ & $q=4$ \\
\hline \multirow{4}{*}{$\begin{array}{c}\text { Units } \\
\text { Purchased }\end{array}$} & $x=1$ & 14 & 10 & 8 & 7 \\
\hline & $x=2$ & 8 & 16 & 12 & 11 \\
\hline & $x=3$ & 4 & 10 & 18 & 14 \\
\hline & $x=4$ & 1 & 7 & 12 & 20 \\
\hline
\end{tabular}

After the buyer informs the seller about the quantity purchased via the computer, the computer will show the seller and the buyer the quantity purchased, the true quality and their own payoffs for the round just played. For an instance, if the true quality is 4 and the buyer chooses to purchase 3 units, then the seller's payoff is $\$ 12$ and the buyer's payoff is $\$ 14$.

The experiment proceeds to the next round after both the seller and the buyer acknowledge this information by clicking the button on the computer screen. In the next round, each participant again is randomly selected to be a buyer or a seller and randomly matched with some participant of the experiment to play the market game. The true quality of the seller in this market game is also randomly selected and may differ from the true quality of the prior round. Phase 1 ends after 30 rounds of the market game have been played.

\section{Phase 2}

Phase 2 consists of a simple test. The test is made up of 30 questions. For every question, there is a pattern with a piece missing and a number of pieces below the pattern. You have to choose which of the pieces below is the missing one to complete the pattern. For each question, one and only one of these pieces is the missing one to complete the pattern. You will score 1 point for every correct answer. After completing the test, you will be informed of your own test score. The test score will not affect your payment that you receive from the experiment. 
After completing both phases 1 and 2, your cash payment will be displayed on your computer screen. Your cash payment will be your payoff from one round randomly drawn from the 30 rounds of the market game plus a $\$ 5$ show-up fee.

\section{Phase 3}

While waiting to be called upon for payment, please complete the questionnaire that the experimenter will hand you. The questionnaire contains questions about demographics. Please carefully complete this questionnaire as this information is very important to us. After completing the questionnaire, please remain in your seat until you have been called upon for payment.

Thank you very much for your participation. 


\section{Screenshots}

Figure 16: Seller's Message Options

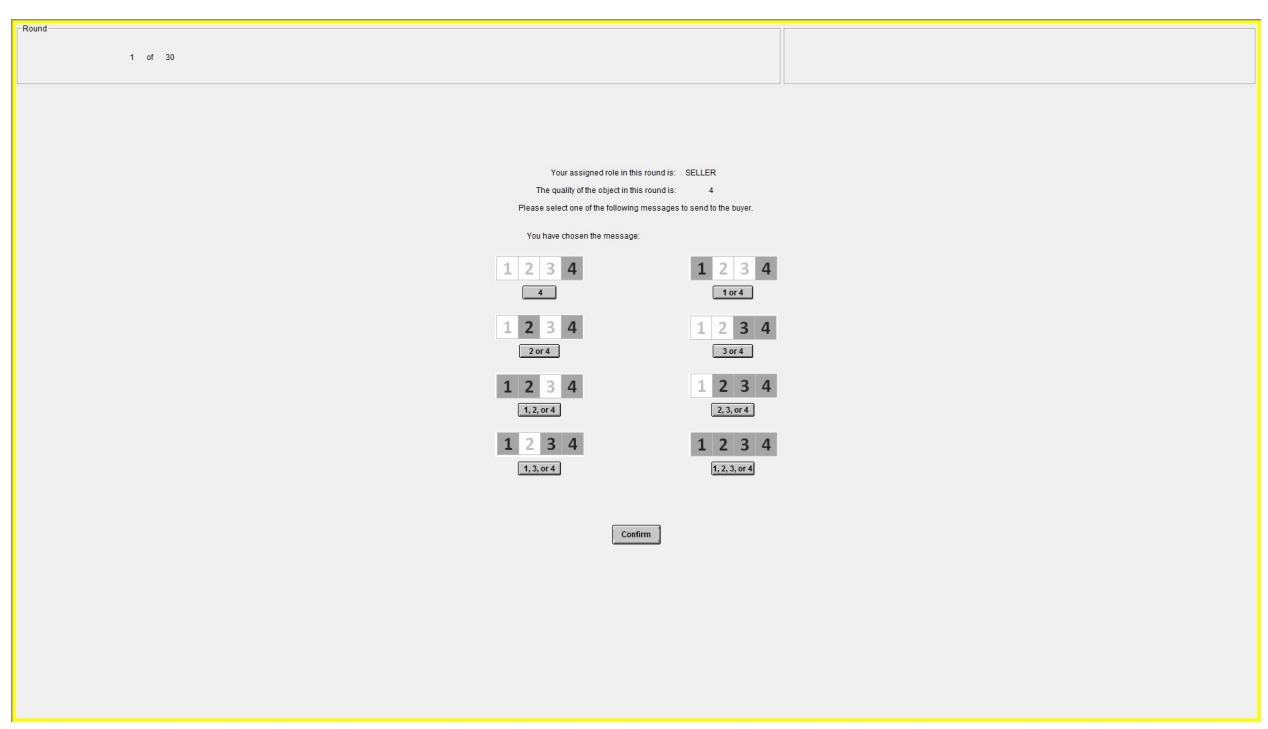

Figure 17: Buyer's Purchase Decision

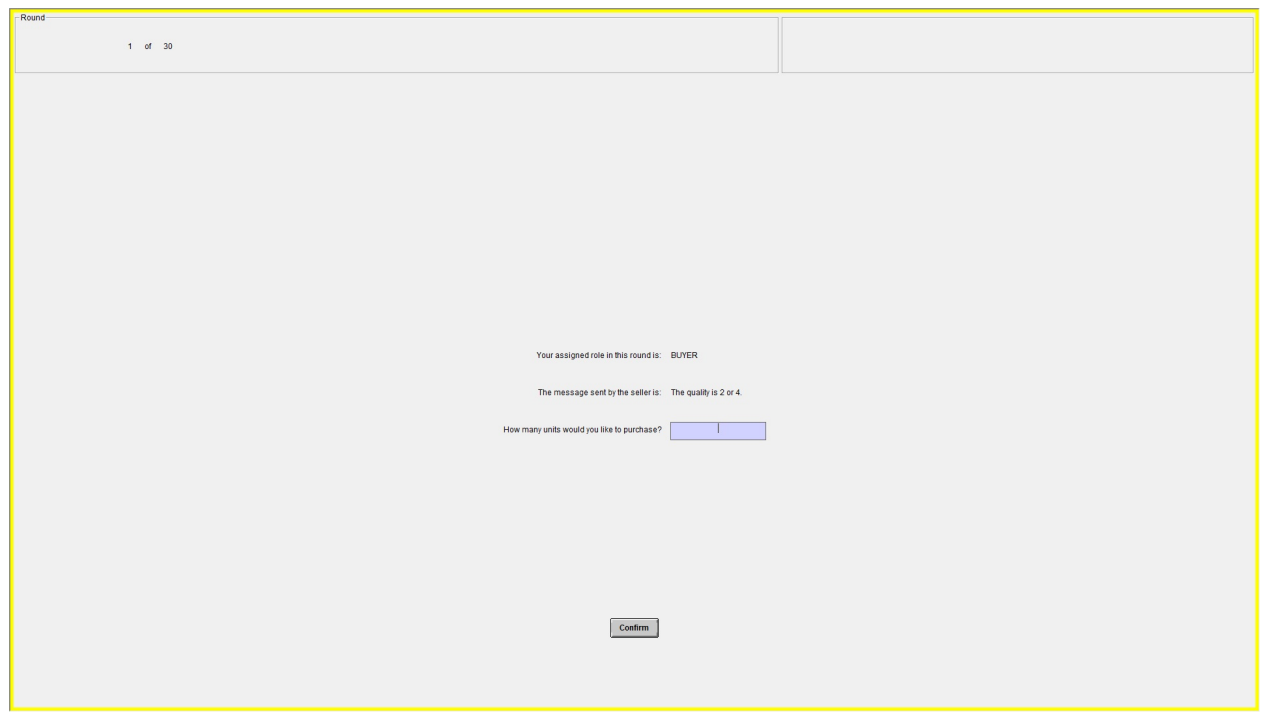


Figure 18: Seller's Payoff Information at the End of Each Round

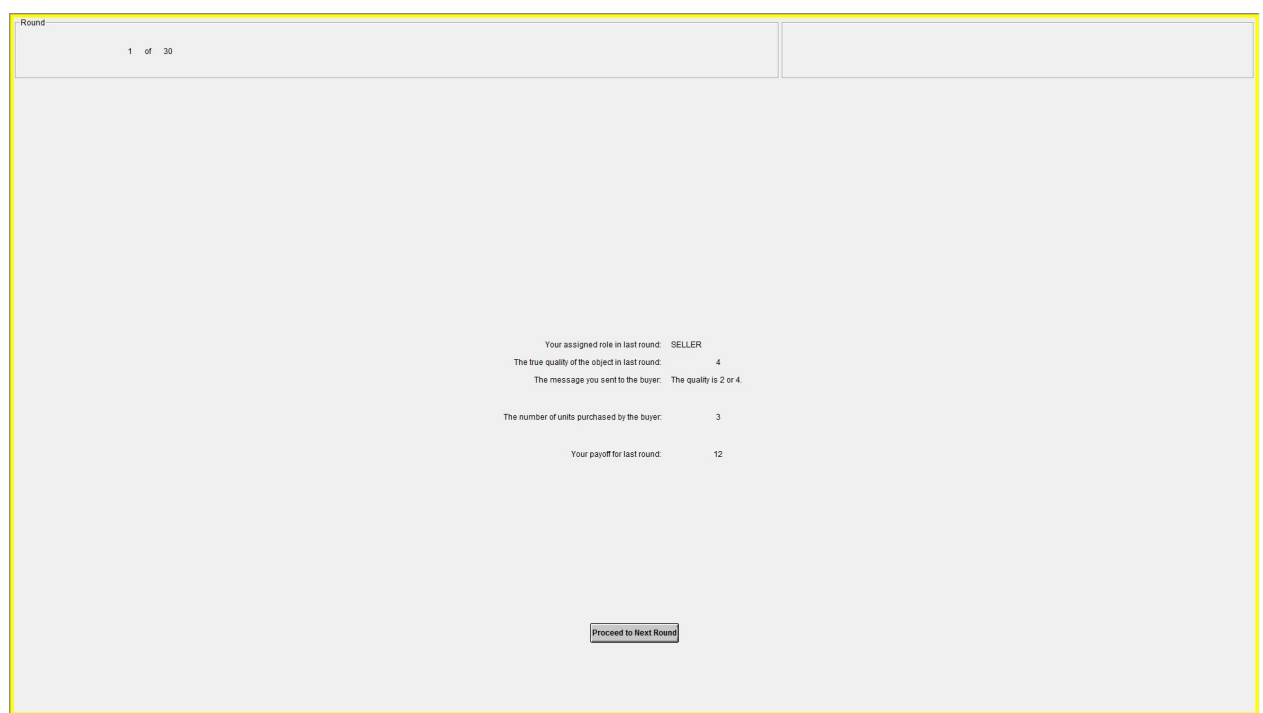

Figure 19: Buyer's Payoff Information at the End of Each Round

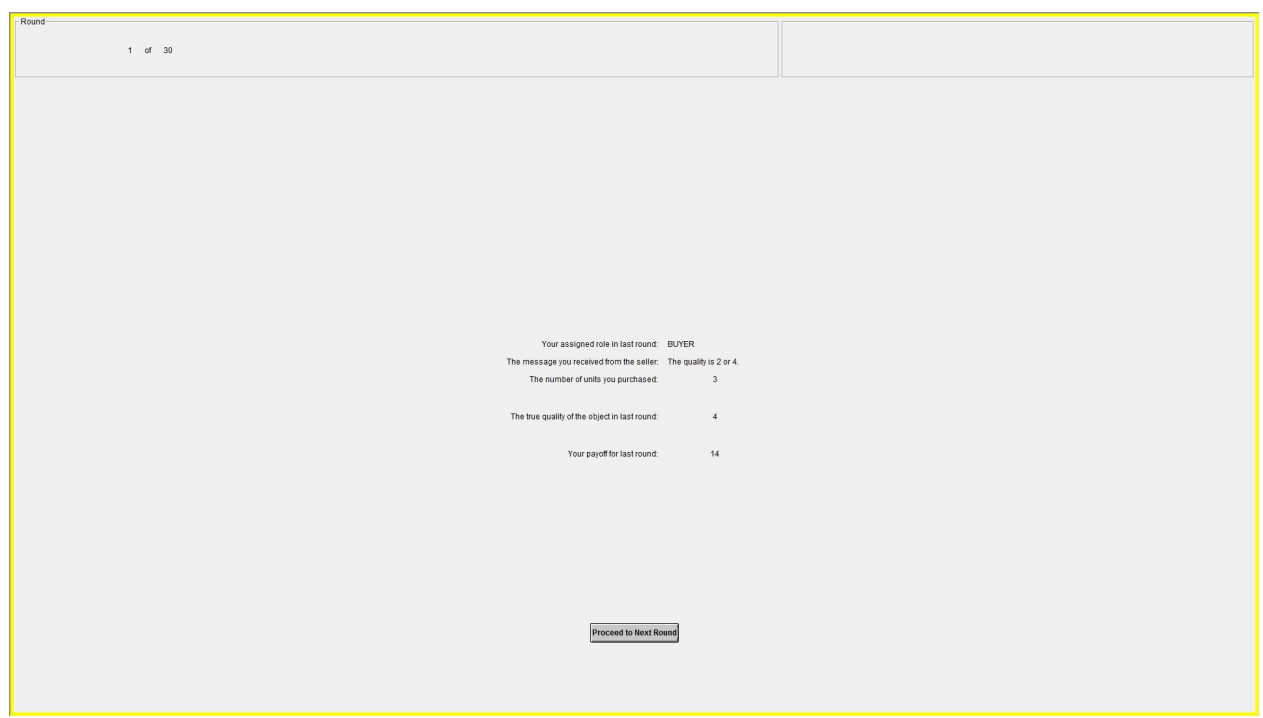

\section{E Questionnaire}


1. What is your gender?

$\square$ Male $\square$ Female

2. What is your primary racial background?

$\square$ White $\square$ Black or African $\square$ Native American $\square$ Asian

$\square$ Hawaiian or other Pacific Islander $\square$ Mixed or other

3. What language(s) do you speak?

4. How fluent are you in English?

$\square$ Native $\square$ Fluent $\square$ Basic $\square$ Least

5. What academic year are you currently in?

$\square 1^{\text {st }} \square 2^{\text {nd }} \square 3^{\text {rd }} \square 4^{\text {th }} \square 5^{\text {th }} \square 6^{\text {th }} \square$ Graduate

6. What is (are) your major(s)?

7. What is your GPA?

8. Have you taken any course on the game theory?

$\square$ Yes $\square$ No

9. Have you taken any course in microeconomics? $\square$ Yes $\square$ No

10. Have you taken any course in decision making? $\square$ Yes $\square$ No

11. How old are you (in years)?

12. Have you participated in an economics experiment before? $\square$ Yes $\square$ No

13. Did you find this experiment fun to play? $\square$ Yes $\square$ No

14. Did you have difficulties in understanding the experiment? $\square$ Yes $\square$ No

15. Are you interested in playing chess or other similar strategy games? $\square$ Yes $\square$ No

16. How do you see yourself? Are you generally a person who is fully prepared to take risks or do you try to avoid taking risks? Please check a box on below scale, where the value 0 means 'not at all willing to take risks' and the value 10 means 'very willing to take risks'.

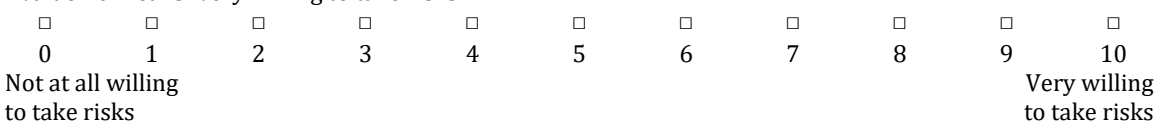

17. How do you see yourself? Are you generally a person who embraces novel things or do you hesitate to adopt novelties? Please check a box on the scale, where the value 0 means 'not at all willing to adopt novelties' and the value 10 means 'very willing to adopt novelties'.

$\begin{array}{ccccccccccc}\square & \square & \square & \square & \square & \square & \square & \square & \square & \square & \square \\ 0 & 1 & 2 & 3 & 4 & 5 & 6 & 7 & 8 & 9 \\ \text { Not at all willing } & & & & & & & & & \text { Very willing to } \\ \text { to adopt novelties } & & & & & & & & & & \text { adopt novelties }\end{array}$

\section{F Identification of Levels}



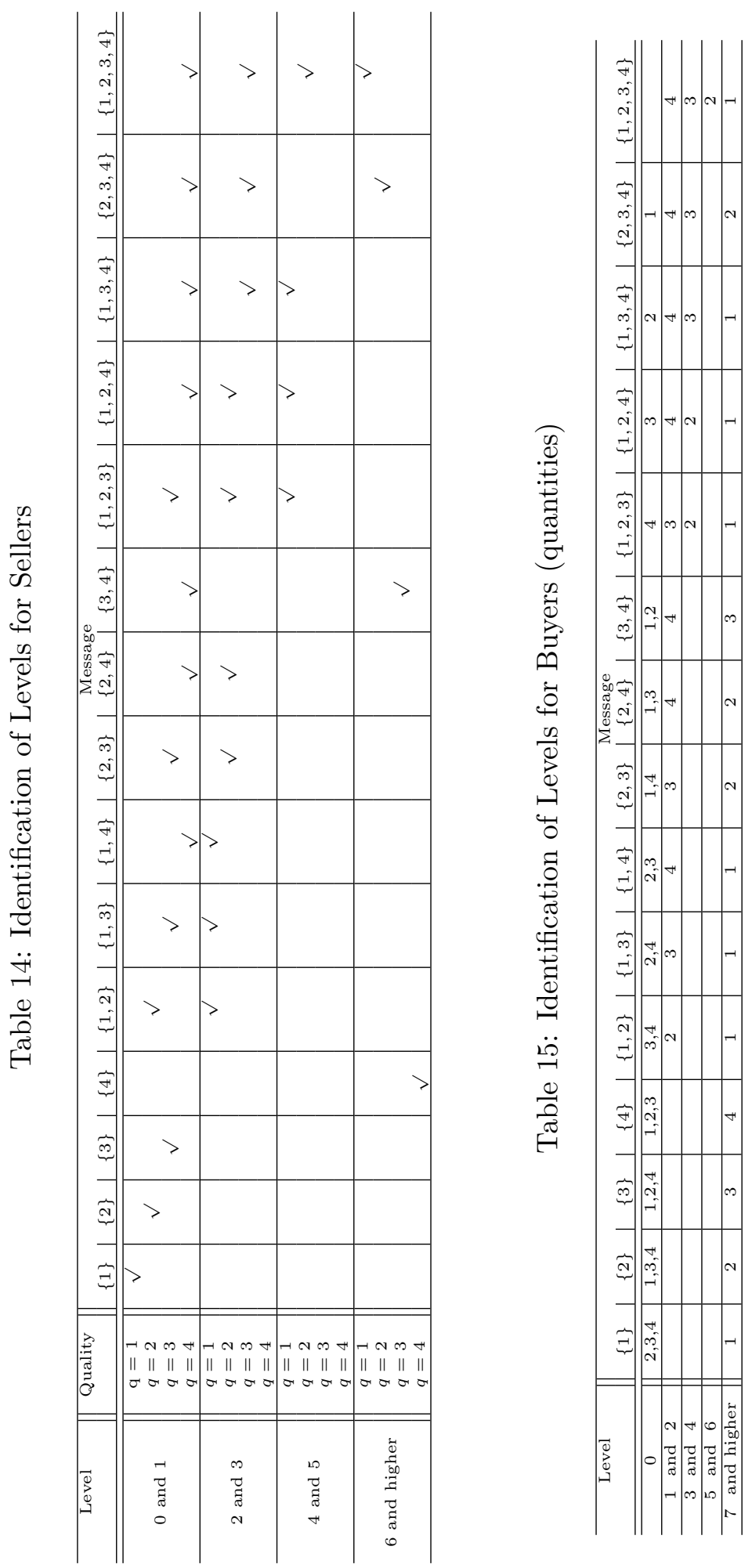


\section{G Distributions of Levels}

Table 16: Distribution of Levels for Sellers

\begin{tabular}{c||c|c|c}
\hline \multicolumn{1}{c||}{ Level } & \multicolumn{2}{c|}{ Treatment 4-4 } & Treatment 2-4 \\
& Round 1-15 & Round 16-30 & Round 16-30 \\
\hline \hline 0 and 1 & 26.88 & 20.97 & 28.49 \\
2 and 3 & 30.65 & 30.65 & 21.51 \\
4 and 5 & 18.28 & 15.59 & 16.13 \\
6 and higher & 24.19 & 32.80 & 33.87 \\
\hline
\end{tabular}

Table 17: Distribution of Levels for Buyers

\begin{tabular}{c||c|c|c}
\hline \multicolumn{1}{c||}{ Level } & \multicolumn{2}{|c}{ Treatment 4-4 } & Treatment 2-4 \\
& Round 1-15 & Round 16-30 & Round 16-30 \\
\hline \hline 0 & 3.76 & 1.61 & 6.45 \\
1 and 2 & 13.98 & 4.84 & 12.90 \\
3 and 4 & 20.97 & 8.60 & 17.20 \\
5 and 6 & 7.53 & 1.08 & 5.91 \\
7 and higher & 53.76 & 83.87 & 57.53 \\
\hline
\end{tabular}

\section{References}

[1] Alaoui, L. and A. Penta (2017). Endogenous depth of reasoning. Review of Economic Studies 83, 1297-1333.

[2] Arad, A. and A. Rubinstein (2012). The 11-20 money request game: A level- $k$ reasoning study. American Economic Review 102, 3561-3573.

[3] Battigalli, P. (2006). Rationalization in signaling games: Theory and applications. International Game Theory Review 8, 67-93.

[4] Battigalli, P. (1996). Strategic rationality orderings and the best rationalization principle. Games and Economic Behavior 13, 178-200.

[5] Battigalli, P. and A. Friedenberg (2012). Forward induction reasoning revisited, Theoretical Economics 7, 57-98.

[6] Benito-Ostolaza, J.M., Hernández, P., and J.A. Sanchis-Llopis (2016). Do individuals with higher cognitive ability play more stategically? Journal of Behavioral and Experimental Economics 64, 5-11.

[7] Benndorf, V., Kübler, D., and H.-T. Normann (2017). Depth of reasoning and information revelation: An experiment on the distribution of $k$-levels. International Game Theory Review 19, 1750021. 
[8] Benndorf, V., Kübler, D., and H.-T. Normann (2015). Privacy concerns, voluntary disclosure of information, and unraveling: An experiment. European Economic Review 75, $43-59$.

[9] Bhatt, M. and C. Camerer (2005). Self-referential thinking and equilibrium as states of mind in games: FMRI evidence. Games and Economic Behavior 52, 424-459.

[10] Brañas-Garza, P., García-Muñoz, T., and R. Hernán González (2012). Cognitive effort in the beauty contest game. Journal of Economic Behavior and Organization 83, 254-260.

[11] Brandenburger, A., Danieli, A., and A. Friedenberg (2019). Identification of reasoning about rationality, University of Arizona.

[12] Brandenburger, A. and A. Friedenberg (2007). The relationship between rationality on the matrix and the tree. mimeo.

[13] Burks, S.V., Carpenter, J.P., Goette, L., and A. Rustichini (2009). Cognitive skills affect economic preferences, strategic behavior, and job attachment. Proceedings of the National Academy of Sciences, 106, 7745-7750.

[14] Burnham, T.C., Cesarini, D., Johannesson, M., Lichtenstein, P., and B. Wallace (2009). Higher cognitive ability is associated with lower entries in a p-beauty contest. Journal of Economic Behavior and Organization 72, 171-175.

[15] Camerer, C.F., Ho, T.-H., and J.-K. Chong (2004). A cognitive hierarchy model of games. Quarterly Journal of Economics 119, 861-898.

[16] Catonini, E. and N. De Vito (2018). Cautious belief and iterated admissibility, mimeo.

[17] Catonini, E. and N. De Vito (2019). Weak belief and permissibility, mimeo.

[18] Charness, G., Rustichini, A., and J. van de Ven (2011). Self-confidence and strategic deterrence. Mimeo, UCSB.

[19] Cooper, D.J. and J.H. Kagel (2009). The role of context and team play in cross-game learning, Journal of the European Economic Association 7, 1101-1139.

[20] Cooper, D.J. and J.H. Kagel (2008). Learning and transfer in signaling games, Economic Theory 34, 415-439.

[21] Coricelli, G. and R. Nagel (2009). Neural correlates of depth of strategic reasoning in medial prefrontal cortex. Proceedings of the National Academy of Sciences 106, 9163-9168.

[22] Costa-Gomes, M.A. and V.P. Crawford (2006). Cognition and behavior in two-person guessing games: An experimental study. American Economic Review 96, 1737-1768.

[23] Costa-Gomes, M.A., Crawford, V.P., and B. Broseta (2001). Cognition and behavior in normal-form games: An experimental study. Econometrica 69, 1193-1235.

[24] Crawford, V.P. (2003). Lying for strategic advantage: Rational and boundedly rational misrepresentation of intentions. American Economic Review 93, 133-149. 
[25] Crawford, V.P., Costa-Gomes, M.A., and N. Iriberri (2013). Structural models of nonequilibrium strategic thinking: theory, evidence, and applications. Journal of Economic Literature 51, 5-62.

[26] Dekel, E., Friedenberg, A. and M. Siniscalchi (2016). Lexicographic beliefs and assumption, Journal of Economic Theory 163, 955-985.

[27] Deversi, M., Ispano, A., and P. Schwardmann (2019). Spin doctors: A model and an experimental investigation of vague disclosure, University of Munich.

[28] Dufwenberg, M., Sunderam, R., and D.J. Butler (2010). Ephiphany in the game of 21, Journal of Economic Behavior and Organization 75, 132-143.

[29] Fischbacher, Urs (2007). z-Tree: Zurich toolbox for ready-made economic experiments. Experimental Economics 10, 171-178.

[30] Forsythe, R., Isaac, M., and T.R. Palfrey (1989). Theories and tests of "blind bidding" in sealed-bid auctions. RAND Journal of Economics 20, 214-238.

[31] Goerganas, S., Healy, P.J., and R. A. Weber (2015). The persistency of strategic sophistication. Journal of Eonomic Theory 159, 369-400.

[32] Gill, D. and V. Prowse (2016). Cognitive ability, character skills, and learning to play equilibrium: a level- $k$ analysis. Journal of Political Economy 124, 1619-1676.

[33] Greiner, B. (2004). The online recruitment system ORSEE - a guide for the organization of experiments in economics. Papers on Strategic Interaction 2003-10, Max Planck Institute of Economics, Strategic Interaction Group.

[34] Grossman, S.J. (1981). The informational role of warranties and private disclosure of product quality. Journal of Law and Economics 24, 461-483.

[35] Grossman, S.J. and O. Hart (1980). Disclosure laws and takeover bids. Journal of Finance $35,323-334$.

[36] Hagenbach, J. and E. Perez-Richet (2018). Communication with evidence in the lab, Games and Economic Behavior 112, 139-165.

[37] Heifetz, A., Meier, M., and B.C. Schipper (2019a). Prudent rationalizability in generalized extensive-form games with unawareness, B.E. Journal of Theoretical Economics, forthcoming.

[38] Heifetz, A., Meier, M., and B.C. Schipper (2019b). Comprehensive rationalizability, Games and Economic Behavior 116, 185-202.

[39] Ho, T.-H. and X. Su (2013). A dynamic level- $k$ model in sequential games. Management Science 59, 452-469.

[40] Jin, G.Z., Luca, M., and D. Martin (2019a). Is no news (perceived as) bad news? An experimental investigation of information disclosure. Working paper. 
[41] Jin, G.Z., Luca, M., and D. Martin (2019b). Complex disclosure. Working paper.

[42] Keisler, J. and Lee B.S. (2015). Common assumption of rationality, mimeo.

[43] King, R.R., and D.E. Wallin (1991). Market-induced information disclosure: An experimental markets investigation. Contemporary Accounting Research 8, 170-197.

[44] Kneeland, T. (2015). Identifying higher-order rationality, Econometrica 83, 2065-2079.

[45] Lee B.S. (2016). Admissibility and assumption, Journal of Economic Theory 163, 42-72.

[46] Li, Y.X. and B.C. Schipper (2018). Disclosure under unawareness: An Experiment. University of California, Davis.

[47] Meier, M. and B.C. Schipper (2012). Conditional dominance in games with unawareness. University of California, Davis.

[48] Milgrom, P. (2008). What the seller won't tell you: Persuasion and disclosure in markets. Journal of Economic Perspectives 22, 115-131.

[49] Milgrom, P. (1981). Good news and bad news: Representation theorems and applications. The Bell Journal of Economics 12, 380-391.

[50] Milgrom, P. and J. Roberts (1986). Relying on the information of interested parties. Rand Journal of Economics 17, 18-32.

[51] Nagel, R. (1995). Unraveling in guessing games: An experimental study. American Economic Review 85, 1313-1326.

[52] Oechssler, J., Roider, A., and P.W. Schmitz (2009). Cognitive abilities and behavioral biases. Journal of Economic Behavior and Organization 72, 147-152.

[53] Pearce, D.G. (1984). Rationalizable strategic behavior and the problem of perfection. Econometrica 52, 1029-1050.

[54] Raven, J. (2008). General introduction and overview: The Raven progressive matrices tests: Their theoretical basis and measurement model, in: Raven, J. and J. Raven (Eds.), Uses and abuses of intelligence: Studies advancing Spearman and Ravens quest for nonarbitrary metrics. Unionville, New York: Royal Fireworks Press, 17-68.

[55] Raven, J., Raven, J.C., and J.H. Court (2000). Manual for Raven's Progressive Matrices and Vocabulary Scales. San Antonio, TX: Pearson.

[56] Schipper, B.C. and H.Y. Woo (2019). Political awareness, microtargeting of voters, and negative electoral campaigning. Quarterly Journal of Political Science 14, 41-88.

[57] Schipper, B.C. and H. Zhou (2019). Extensive-form level- $k$ thinking, University of California, Davis.

[58] Shimoji, M. and J. Watson (1998). Conditional dominance, rationalizability, and game forms. Journal of Economic Theory 83, 161-195. 
[59] Stahl, D.O. and P.W. Wilson (1995). On players' models of other players: Theory and experimental evidence. Games and Economic Behavior 10, 218-254.

[60] Yang C.-C. (2015). Weak assumption and iterative admissibility, Journal of Economic Theory 158, 87-101. 